\title{
High-temperature effects in hypersonic flows
}

\author{
DINESH K PRABHU
}

Computational and Theoretical Fluid Dynamics Division, National Aerospace Laboratories, Bangalore 560017, India

MS received 31 August 1994; revised 21 January 1995

\begin{abstract}
The hypersonic $(M=25)$ flow past a $10^{\circ}$ right circular cone at $0^{\circ}$ angle of attack has been computed using an implicit, noniterative, finitedifference scheme for the Parabolized Navier-Stokes (PNS) equations. Three different gas models have been considered in the study - (1) ideal gas $(\gamma=$ constant $)$, (2) equilibrium air, and (3) five species $\left(\mathrm{N}_{2}, \mathrm{O}_{2}, \mathrm{NO}, \mathrm{N}, \mathrm{O}\right)$ chemical nonequilibrium air. For the nonequilibrium air case, three different models have been considered - (a) one in which the constituent species have no internal structure (i.e., species are ideal gases), (b) one in which the constituent species have internal structure (i.e., species are thermally perfect gases), and (c) one in which the species have internal structure and their thermodynamic properties have been obtained from quantum mechanical and spectroscopic calculations.
\end{abstract}

Keywords. Hypersonic flows; chemical equilibrium/nonequilibrium flows; planar/axisymmetric flows; finite-difference formulation.

\section{Introduction}

Research in the area of hypersonic aerothermodynamics in India has received a great impetus from the success of the Agni re-entry vehicle (especially from the point of designing its heat shield). In addition to the Agni programme, there are also plans for a one-stage-to-orbit Hyperplane whose design will require the integration of the aerothermodynamic and propulsive (supersonic combustion of $\mathrm{H}_{2}$ and air) aspects of flight. The development, design and testing of such vehicles and perhaps future space vehicles will require ground-based experimental facilities capable of simulating the extreme conditions normally encountered at hypersonic speeds. Ground-based test facilities are expensive from an operational point of view and the ones currently available in India are inadequate. This handicap can be overcome to a large extent through the use of high-speed digital computers that are widely available today.

It is conventional to classify any flow whose Mach number exceeds 5 as hypersonic flow. However, this is merely a rule of thumb and is insufficient (Anderson 1989). Hypersonic flow is markedly different from the usual supersonic flow. For instance, the 
elevated temperatures (due to high enthalpies) in the hypersonic viscous shock layer result in the occurrence of several complex physical phenomena such as vibrational relaxation, chemical reactions, ionisation, radiation, etc. which are not present in conventional supersonic flows. These phenomena are generally termed as "realgas effects" ("high-temperature effects" is a more appropriate term) and are usually rate processes (mostly due to atomic, molecular, electronic or photonic collisions) which could be either fast or slow compared to the characteristic flow time. On the one hand, if the rate processes are infinitely fast, especially in the denser reaches of the atmosphere, then one has a local thermodynamic equilibrium situation. For this case, the local thermodynamic state is defined uniquely by any two-state variables such as the internal energy and density (two commonly used dependent variables in CFD). The usual Navier-Stokes equations expressing the conservation of mass, linear momentum, and energy with suitable modifications to the equation of state are enough to model the flow. On the other hand, if the rate processes occur at finite rates, especially in the rarer reaches of the atmosphere, then one has a nonequilibrium situation. For this case, apart from the usual Navier-Stokes equations, one must consider the equations governing the rate processes (Anderson 1989; Park 1990). For instance, if chemical reactions occur at finite rates, then one has to take into consideration the conservation of mass of each of the species that makes up the gas mixture. This implies that the governing equation set is expanded (considerably if one has a large number of species). In addition to these phenomena, one must also take into account the internal structures of the species that compose the flow stream. As a first approximation, one can assume the atomic species to be point masses and the molecular species to be rigid rotors. For a more rigorous analysis, however, one must account for the realistic structure of the atomic and molecular species.

Once the flow has been modelled mathematically, the equations governing the flow must be solved subject to some boundary conditions. The usual approach is to solve the equations numerically since they are intractable to analytical solution. There are several approaches that are possible (Anderson et al 1982), (1) solve the time-dependent form of the equations iteratively till a steady-state solution (usually the one of interest) is reached, (2) solve the time-independent form of the equations by means of space marching along the dominant flow direction, or (3) a combination of the two. The first approach is usually resource intensive for the three-dimensional case, both in terms of computer memory and time. The second approach reduces the dimensionality of the problem by one, i.e., some initial solution surface is marched in the dominant flow direction (time-like axis). This approach is subject to some restrictions (for example, the inviscid flow must be everywhere supersonic), but in the cases where such an approach is viable, it provides solutions of accuracy comparable to that obtained from the first approach and is also substantially less resource intensive. Some examples of the space-marching method are the Viscous Shock Layer (VSL) method, and the Parabolised Navier-Stokes (PNS) method (Rubin \& Tannehill 1992) etc.

The PNS equations are obtained from the time-dependent Navier-Stokes equations by dropping the time terms and the viscous terms in the streamwise direction. The equations so obtained are actually hyperbolic-elliptic due to the presence of the subsonic layer near the wall. This layer permits pressure disturbances to travel upstream (upstream influence) resulting in exponentially growing solutions (departure solutions). Consequently, the equations are not well posed for a space-marching method. There are several ways of overcoming this difficulty; the ones due to Vigneron 
et al (1978) or Schiff \& Steger (1979) are commonly used. In the Schiff and Steger method, the pressure at the sonic point within the boundary layer is imposed in the direction normal to the body throughout the subsonic zone. In the technique due to Vigneron et al (1978), only a fraction of the streamwise pressure gradient is retained in the subsonic boundary layer. This fraction, which is a function of the local streamwise Mach number, is obtained from an eigenvalue analysis of the governing equations. The eigenvalue analysis also reveals that the PNS equations cannot be used in flows where the inviscid Mach number is less than 1, e.g., the nose of a blunt body which has a large embedded subsonic zone in the shock layer. In conjunction with time-dependent Navier-Stokes equations, PNS methods have been successfully used by many researchers for a variety of problems ranging from three-dimensional ideal supersonic flow to three-dimensional reacting hypersonic flow (Rakich et al 1984; Prabhu \& Tannehill 1986; Prabhu 1987, 1993; Prabhu et al 1988, 1993).

After the choice of time or space-marching method has been made, one has a choice of several numerical algorithms - (i) explicit methods, (ii) implicit methods, or (iii) hybrid explicit-implicit methods (Anderson et al 1982). Explicit methods are usually slow since they are limited by the "effective time scale" of the problem in addition to the CFL limit. Implicit methods, however, do not normally have CFL limitations and can resolve multiple scales within the problem but require a lot more computer resources and time. The choice of method is actually dictated by the problem. For instance, unsteady nonequilibrium flows require the use of explicit methods to capture both the flow time scale and the reaction time scale (Cambier et al 1992). For a steady equilibrating flow, where the reaction time scale is much less than the flow time scale, an implicit method is more appropriate (Cambier \& Prabhu 1992).

Other considerations in choosing the numerical scheme are the choice of finitedifference or finite-volume method and the representation of the numerical flux. Until recently, the finite-difference method was used with a symmetric representation of fluxes, i.e., central differences. With advances in numerical analysis, the choice has since shifted to finite-volume methods with biased representation of numerical fluxes, i.e., upwind differencing.

In the present work, a finite-difference method with central-differencing of fluxes is chosen to solve the PNS equations for steady, reacting/nonreacting, laminar, hypersonic flow past a simple geometry such as a right circular cone. The primary objectives of the study are:

- To bring out the salient features of hypersonic viscous shock layers through the study of high-temperature effects such as chemical reactions.

- To study the influence of the grid on the computed solution.

- To study the influence of various gas models (ideal, equilibrium, and nonequilibrium air) on the computed solution.

- To provide a comprehensive set of "benchmark" results for code validation.

With these objectives in mind, a two-dimensional planar/axisymmetric PNS code has been developed. The code is based on an implicit, finite-difference method with central differencing of inviscid/viscous fluxes. Three types of gas models have been included in the code - (1) ideal gas $(\gamma=1.4)$, (2) equilibrium air whose thermodynamic and transport properties are based on piecewise continuous curve fits developed by Srinivasan et al (1987a, b), and (3) nonequilibrium air consisting of five species $-\mathrm{N}_{2}$, $\mathrm{O}_{2}$, NO, N, and $\mathrm{O}$. For the last case, three further sub-cases are considered - (a) the 


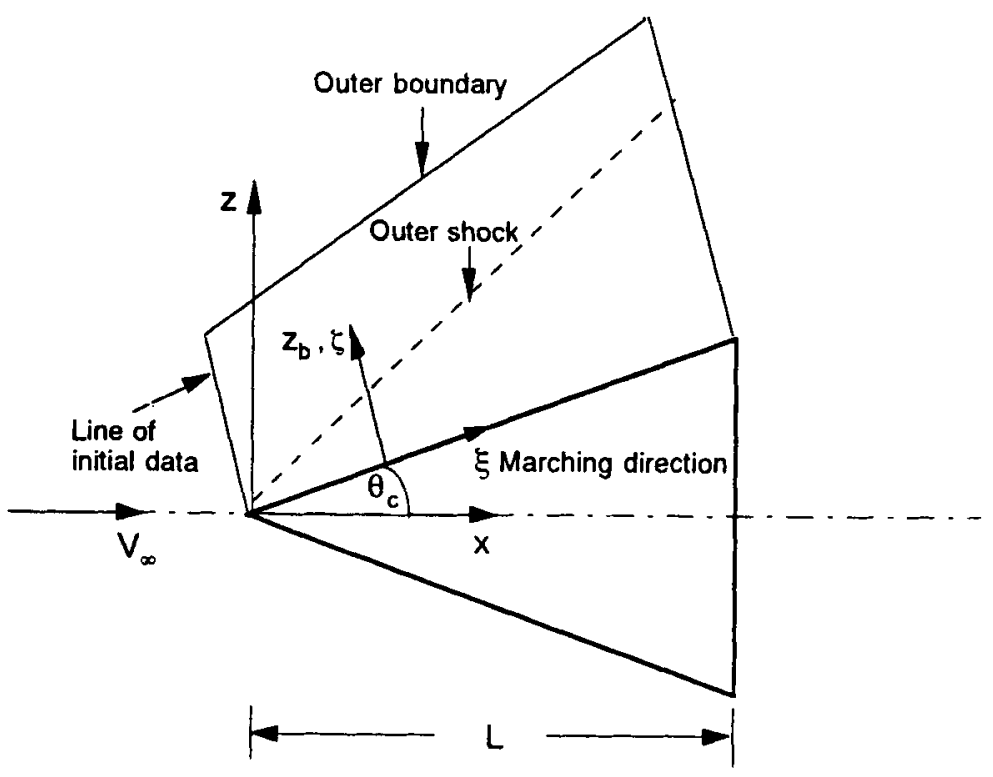

Figure 1. Coordinate system used in the present study. $\xi, \zeta$ are generalised coordinates (not necessarily orthogonal). $L$ is the characteristic length. The outer boundary is placed far enough from the body to be able to capture the outer shock.

constituent species have no internal structure, (b) the constituent species have internal structure and their thermodynamic properties are obtained from Blottner et al (1971), and (c) the constituent gases possess internal structure and their thermodynamic properties are obtained from accurate spectroscopic constants and potentials (Liu \& Vinokur 1989).

\section{Governing equations and boundary conditions}

\subsection{Ideal/Equilibrium gas}

The equations governing the steady, two-dimensional planar/axisymmetric, laminar flow of an ideal/equilibrium gas can be written for a general curvilinear coordinate system $(\xi, \zeta)$ shown in figure 1 as:

$$
\frac{\partial}{\partial \xi}\left(\mathbf{E}^{i}\right)+\frac{\partial}{\partial \zeta}\left(\mathbf{G}^{i}-\mathbf{G}^{v}\right)=j\left(\mathbf{S}^{i}-\mathbf{S}^{v}\right)
$$

where $j=0$ for planar flow, and $j=1$ for axisymmetric flow. The superscripts $i$ and $v$ represent the inviscid and viscous quantities, respectively. The coordinate $\xi$ is identified as the primary or streamwise coordinate and $\zeta$ as the normal coordinate.

The inviscid flux vectors, $\mathbf{E}^{i}$ and $\mathbf{G}^{i}$, are given by

$$
\mathbf{E}^{i}=\frac{1}{J}\left\{\begin{array}{c}
\rho \hat{U} \\
\rho u \hat{U}+\xi_{x} p \\
\rho w \hat{U}+\xi_{z} p \\
\left(\rho e_{t}+p\right) \hat{U}
\end{array}\right\}, \quad \mathbf{G}^{i}=\frac{1}{J}\left(\begin{array}{c}
\rho \hat{W} \\
\rho u \hat{W}+\zeta_{x} p \\
\rho w \hat{W}+\zeta_{z} p \\
\left(\rho e_{t}+p\right) \hat{W}
\end{array}\right)
$$


where $\rho\left(\mathrm{kg} / \mathrm{m}^{3}\right)$ is the mass density of the gas, $p(\mathrm{~Pa})$ is the static pressure, $u, w(\mathrm{~m} / \mathrm{s})$ are the Cartesian components of velocity, and $e_{t}(\mathrm{~J} / \mathrm{kg})$ is the specific total energy given by:

$$
e_{t}=e+\frac{1}{2}\left(u^{2}+w^{2}\right)
$$

where $e(\mathrm{~J} / \mathrm{kg})$ is the specific internal energy of the gas.

The viscous flux vector, $\mathbf{G}^{\boldsymbol{v}}$, is given by

$$
\mathbf{G}^{v}=\frac{1}{J}\left(\begin{array}{c}
0 \\
\mu\left(l_{1} u_{\zeta}+l_{3} w_{\zeta}\right) \\
\mu\left(l_{3} u_{\zeta}+l_{2} w_{\zeta}\right) \\
\mu\left[\left(l_{1} u+l_{3} w\right) u_{\zeta}+\left(l_{3} u+l_{2} w\right) w_{\zeta}\right]+\kappa l_{4} T_{\zeta}
\end{array}\right),
$$

where $\mu(\mathrm{Pa} \cdot \mathrm{s})$ is the coefficient of viscosity and $\kappa(\mathrm{W} / \mathrm{m} \cdot \mathrm{K})$ is the coefficient of thermal conductivity. The subscript $\zeta$ indicates partial differentiation w.r.t. the coordinate $\zeta$. The coefficients $l_{1}, \ldots, l_{4}$ are given by

$$
l_{1}=\frac{4}{3} \zeta_{x}^{2}+\zeta_{z}^{2}, \quad l_{2}=\zeta_{x}^{2}+\frac{4}{3} \zeta_{z}^{2}, \quad l_{3}=\frac{1}{3} \zeta_{x} \zeta_{z}, \quad l_{4}=\zeta_{x}^{2}+\zeta_{z}^{2},
$$

$\xi_{x}, \xi_{z}, \zeta_{x}, \zeta_{z}$ are the metrics and $J$ is Jacobian of the coordinate transformation $[(x, y) \rightarrow(\xi, \zeta)] . \hat{U}$ and $\hat{W}$ are the contravariant components of velocity and are given by

$$
\hat{U}=\xi_{x} u+\xi_{z} w, \quad \hat{W}=\zeta_{x} u+\zeta_{z} w .
$$

Note that the viscous flux contains terms in the $\zeta$ direction only, i.e., the streamwise

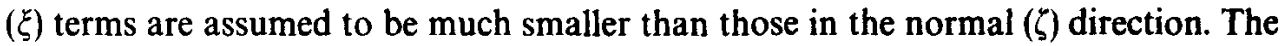
equations are thus said to be "parabolized". The governing equations, however, are still hyperbolic-elliptic and hence improperly posed for space-marching solutions. The conditions for well-posedness are elaborated on in a later section.

The vectors $\mathbf{S}^{i}$ and $\mathbf{S}^{v}$ are source terms for the axisymmetric formulation and are not given here.

For the case of the ideal/equilibrium gas, the vector of conservation variables $\mathbf{Q}$ is chosen as

$$
\mathbf{Q}=\left\{\rho, \rho u, \rho w, \rho e_{\sharp}\right\}^{T}
$$

\subsection{Nonequilibrium gas}

The chemically reacting gas is actually a mixture of gases that react with one another. The rates of these reactions are finite as compared to the equilibrium gas where the reaction rates are infinitely large. Therefore, in addition to the conservation of mass of the gas mixture as a whole, one has to satisfy the law of conservation of mass for each constituent species.

The equations governing the steady, two-dimensional planar/axisymmetric, laminar flow of a chemically reacting gas consisting of $n$ constituents can be written for a general curvilinear coordinate system $(\xi, \zeta)$ shown in figure 1 as:

$$
\frac{\partial}{\partial \xi}\left(\mathbf{E}^{i}\right)+\frac{\partial}{\partial \zeta}\left(\mathbf{G}^{i}-\mathbf{G}^{v}\right)=j\left(\mathbf{S}^{i}-\mathbf{S}^{v}\right)+\mathbf{W}^{c},
$$


where the superscript $c$ represents the chemical source terms. The other symbols retain the same meanings as before. The inviscid flux vectors, $\mathbf{E}^{i}$ and $\mathbf{G}^{i}$, are given by

$$
\mathbf{E}^{i}=\frac{1}{J}\left(\begin{array}{c}
\rho \hat{U} \\
\rho u \hat{U}+\xi_{x} p \\
\rho w \hat{U}+\xi_{z} p \\
\rho H \hat{U} \\
\rho c_{1} \hat{U} \\
\rho c_{2} \hat{U} \\
\vdots \\
\rho c_{n-1} \hat{U}
\end{array}\right), \quad \mathbf{G}^{i}=\frac{1}{J}\left(\begin{array}{c}
\rho \hat{W} \\
\rho u \hat{W}+\zeta_{x} p \\
\rho w \hat{W}+\zeta_{z} p \\
\rho H \hat{W} \\
\rho c_{1} \hat{W} \\
\rho c_{2}, \hat{W} \\
\vdots \\
\rho c_{n-1} \hat{W}
\end{array}\right)
$$

where $c_{1}, c_{2}, \ldots, c_{n-1}$ are the mass fractions of the species and $H(\mathrm{~J} / \mathrm{kg})$ is the specific total enthalpy of the mixture. This is given by

$$
H=h+\frac{1}{2}\left(u^{2}+w^{2}\right),
$$

where $h(\mathrm{~J} / \mathrm{kg})$ is the specific static enthalpy of the mixture.

Note that only $n-1$ equations are considered for the conservation of species mass. The $n$th equation has been replaced by the global mass conservation equation and the mass fraction of the $n$th species is obtained from the algebraic relation

$$
c_{n}=1-\sum_{s=1}^{n-1} c_{s} .
$$

Compare the flux vectors given by (2) with those given in (9) - for the nonequilibrium case the flux vectors have $n+3$ components while for the ideal/equilibrium gas case the vectors have 4 components. Therefore, the equation set for a nonequilibrium case can be very large depending on the value of $n$.

The viscous flux vector, $\mathbf{G}^{v}$, is given by

$$
\mathbf{G}^{v}=\frac{\mu}{J}\left|\begin{array}{c}
0 \\
\left(l_{1} u_{\zeta}+l_{3} w_{\zeta}\right) \\
\left(l_{3} u_{\zeta}+l_{2} w_{\zeta}\right) \\
{\left[\left(l_{1} u+l_{3} w\right) u_{\zeta}+\left(l_{3} u+l_{2} w\right) w_{\zeta}\right]+} \\
l_{4}\left(\frac{\kappa}{\mu} T_{\zeta}+\Sigma_{s} h_{s} \frac{\mathscr{D}}{v} c_{s \zeta}\right) \\
\frac{\mathscr{D}}{v} l_{4} c_{1_{\zeta}} \\
\vdots \\
\frac{\mathscr{D}}{v} l_{4} c_{n-1}
\end{array}\right|
$$

where $\mu(\mathrm{Pa} \cdot \mathrm{s})$ is the coefficient of viscosity, $v=\mu / \rho$ is the kinematic viscosity, 
$\kappa(\mathrm{W} / \mathrm{m} \cdot \mathrm{K})$ is the coefficient of thermal conductivity, and $\mathscr{D}\left(\mathrm{m}^{2} / \mathrm{s}\right)$ is the coefficient of binary diffusion. The coefficients $l_{1}, \ldots, l_{4}$ are given by (5).

The chemical source vector, $\mathbf{W}^{c}$ is

$$
W^{c}=\left\{0,0,0,0, \dot{w}_{1}, \dot{w}_{2}, \ldots, \dot{w}_{n-1}\right\}^{\mathrm{T}},
$$

where $\dot{w}_{s}\left(\mathrm{~kg} / \mathrm{m}^{3} \cdot \mathrm{s}\right)$ represents the mass production rate of species $s$.

The vectors $\mathbf{S}^{i}$ and $\mathbf{S}^{v}$ are source terms for the axisymmetric formulation. They are not given here and can be found elsewhere (Prabhu et al 1988).

For the case of the nonequilibrium gas, the vector of conservation variables $\mathbf{Q}$ is chosen as

$$
\mathbf{Q}=\left\{\rho, \rho u, \rho w, \rho H, \rho c_{1}, \rho c_{2}, \ldots, \rho c_{n-1}\right\}^{T}
$$

\subsection{Boundary conditions}

The domain of interest is bounded by two boundaries - (a) the far field boundary and (b) the inner or wall boundary.

At the outer boundary freestream conditions are applied. This means that any discontinuities that arise in the flowfield are "captured" as part of the solution. This is possible due to the strong conservation-law form of the equations.

Since the flow is viscous, "no-slip" boundary conditions are applied at the wall, i.e.,

$$
u=w=0 .
$$

The wall is assumed to be isothermal with a constant wall temperature, i.e.,

$$
T_{w}=\text { constant }
$$

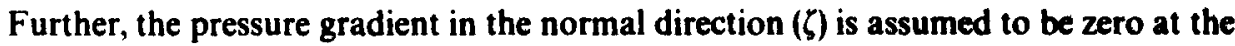
wall. Strictly speaking, one has to solve the normal momentum equation at the wall. In practice, however, this approximation from boundary-layer arguments works well. This boundary condition is expressed mathematically as:

$$
\frac{\partial p}{\partial \zeta}=0
$$

In addition to these boundary conditions, conditions have to be imposed on the species mass densities (or equivalently mass fractions) for the chemical nonequilibrium case. We will assume the wall to be noncatalytic, i.e., the species density gradients normal to the wall are zero. This is expressed as:

$$
\left.\frac{\partial c_{s}}{\partial \zeta}\right|_{w}=0
$$

\section{Gas models}

In order to close the system of governing equations, relations between the thermodynamic quantities and expressions for the transport properties of the gas or gas mixture are required. The relations and expressions depend on the type of gas considered and are discussed in what follows. 


\subsection{Thermodynamic and transport properties: Ideal gas}

The ideal gas obeys the following equations of state (thermal and caloric):

$$
\begin{aligned}
& p=\rho R T, \\
& p=(\gamma-1) \rho e,
\end{aligned}
$$

where $p(\mathrm{~Pa})$ is the static pressure, $\rho\left(\mathrm{kg} / \mathrm{m}^{3}\right)$ is the mass density, $R(\mathrm{~J} / \mathrm{kg} \cdot \mathrm{K})$ is the specific gas constant, $T(\mathrm{~K})$ is the static temperature, $e(\mathrm{~J} / \mathrm{kg})$ the specific internal energy and $\gamma(=\mathrm{a}$ constant $)$ is the ratio of specific heats. The speed of sound $a(\mathrm{~m} / \mathrm{s})$ is defined as

$$
a=(\gamma p / \rho)^{1 / 2} .
$$

The coefficient of viscosity $(\mathrm{Pa} \cdot \mathrm{s})$ is obtained from Sutherland's formula

$$
\mu=\mu_{\text {ref }} T^{3 / 2} /(T+C),
$$

where $\mu_{\text {ref }}$ is a reference viscosity and $C(=110.4 \mathrm{~K})$ is Sutherland's constant. The coefficient of thermal conductivity $(\mathrm{W} / \mathrm{m} \cdot \mathrm{K})$ is obtained from the definition of the Prandtl number

$$
\operatorname{Pr}=\mu C_{p} / \kappa=\text { constant } \Rightarrow \kappa=\mu C_{p} / \operatorname{Pr}
$$

where the specific heat $C_{p}(\mathrm{~J} / \mathrm{kg} \cdot \mathrm{K})$ at constant pressure is given by

$$
C_{p}=\gamma R /(\gamma-1)=\text { constant }
$$

\subsection{Thermodynamic and transport properties: Equilibrium gas}

The equilibrium gas is actually a mixture of gases in which we have local thermodynamic equilibrium. For this case, given any two state variables (for instance $\rho$ and e) we can determine the chemical composition of the gas using Gibbs free-energy minimisation (Gordon \& McBride 1976), or equilibrium constants method (Bailey 1967). Such iterative methods are quite time consuming because the gas composition has to be determined at every grid point at every spatial step. Fortunately, for the special case of air, gas compositions over a wide range of densities and temperatures have already been computed and curve fits for the thermodynamic and transport properties have been obtained (Srinivasan et al 1987a, b). These piecewise continuous curve fits are of adequate accuracy and are briefly described in what follows.

Recall that the primary variables used in the ideal/equilibrium gas formulation are $\rho$ and $e$. For this choice of state variables the curve fits for the thermodynamic properties $p, T$ and $a$ are in functional form (Srinivasan et al 1987a)

$$
p=p(\rho, e) ; \quad a=a(\rho, e) ; \quad T=T(\rho, e) .
$$

We also require the following curve fits for the implementation of boundary conditions (this is explained in a subsequent section):

$$
h=h(p, \rho) ; \quad T=T(p, \rho) .
$$

In addition to these curve fits, we need a link between the fluid dynamics and the equilibrium chemistry. This link is provided by two parameters $\bar{\gamma}$ and $\bar{\beta}$ which are 
defined as

$$
\begin{aligned}
& \bar{\gamma}=h / e, \\
& \bar{\beta}=p / \rho R T .
\end{aligned}
$$

The first parameter is analogous to the usual isentropic exponent $\gamma$ which is the ratio of specific heats. For the case of ideal gas $\bar{\gamma}$ and $\gamma$ are identical. The second parameter is the compressibility of the gas and measures the departure of the gas from its ideal behaviour. For the case of an ideal gas $\bar{\beta}$ is identically 1 .

The first correlation given in (25) is actually obtained from a curve fit of $\bar{\gamma}$,

$$
\bar{\gamma}=\bar{\gamma}(\rho, e),
$$

in conjunction with

$$
p=(\bar{\gamma}-1) \rho e .
$$

Using (30) with the definition of compressibility the temperature can be rewritten as

$$
T=[(\bar{\gamma}-1) / \bar{\beta}](e / R) .
$$

Through the use of (30) and (31), the formulation for the case of equilibrium air is the same as that for ideal gas with $\gamma$ being replaced by $\bar{\gamma}$.

As in the case of the thermodynamic properties, the transport properties are also available in the form of piecewise continuous curve fits (Srinivasan et al 1987b) with $\rho$ and $e$ as the primary variables. The curve fits in functional form are:

$$
\mu=\mu(\rho, e) ; \quad \kappa=\kappa(\rho, e)
$$

\subsection{Thermodynamic and transport properties: Chemical nonequilibrium gas}

In the present context, chemical nonequilibrium gas refers specifically to air consisting of five species $-\mathrm{N}_{2}, \mathrm{O}_{2}, \mathrm{NO}, \mathrm{N}$ and $\mathrm{O}$. These species react with one another and the reactions are assumed to be far from equilibrium, i.e., occur at a finite rate.

We have to consider the properties of the individual species as well as those of the gas mixture. The properties of the individual species depend on the kind of structure the species possess. For instance, we can assume the atomic species to be point masses or take into account their actual quantum structure. These assumptions lead to different values and behaviour of the thermodynamic properties.

The component gases obey the thermal equation of state,

$$
p_{s}=\rho_{s} \mathscr{R} T /\left(\mathscr{M}_{s}\right)
$$

where $p_{s}(\mathrm{~Pa})$ is the partial pressure of species $s, \mathscr{R}$ is the universal gas constant $(8314 \cdot 34 \mathrm{~J} / \mathrm{kmol} \cdot \mathrm{K}), \mathscr{M}_{s}(\mathrm{~kg} / \mathrm{kmol})$ is the molecular mass of species $s$. The caloric equation of state depends on the nature of the constituent species. Three models are considered in the present study. These models differ in the kind of structure possessed by the constituent species of the gas mixture. In the first gas model, the species are assumed to have no internal structure - each atomic species is assumed to be a point mass with three translational degrees of freedom and each diatomic species is assumed to be a rigid rotor with three translational and two rotational degrees of freedom. In other words, the species that constitute the gas mixture are ideal gases. In the other two models, each species has an internal structure - each atomic species has quantised 


\section{IDEAL}

(a)

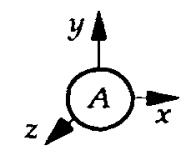

3 Trans. d.o.f. $(f=3)$

(b)

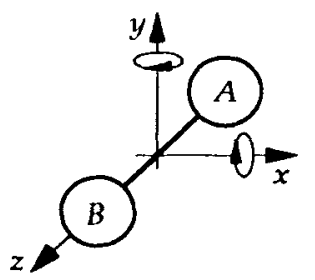

3 Trans. and 2 Rot. d.o.f. $(f=5)$

$$
C_{p}=\frac{f+2}{2} \frac{R}{M}=\text { constant }
$$

REAL

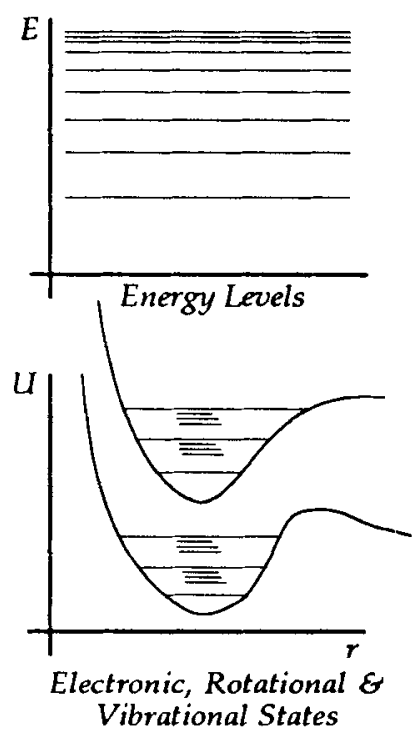

$C_{p}=C_{p}(T)$

Figure 2. Schematic representation of atoms and molecules in the ideal and real senses. Atoms are idealised as point masses (a) while diatomic molecules are idealised as rigid rotors (b). In reality the internal structure is quantised - atoms have discrete energy levels and molecules have electronic, vibrational, and rotational states. The specific heats of the species are constants for the ideal case and functions of temperature for the real case.

energy levels and the diatomic species has quantised electronic states which further contain vibrational and rotational levels. These models are schematically represented in figure 2.

3.3a Mixture of ideal gases: In this model, the atoms are assumed to be point masses with 3 translational degrees of freedom and the diatomic molecules are assumed to be rigid rotors with 3 translation and 2 rotational degrees of freedom (vibrational degrees of freedom being neglected). The specific heat and enthalpy of each individual species are given by

$$
\begin{aligned}
C_{p, s} & =[(f+2) / 2]\left(\mathscr{R} / \mathscr{M}_{s}\right)=\text { constant } \\
h_{s} & =C_{p, s} T+h_{s}^{0},
\end{aligned}
$$

where $h_{s}^{0}(\mathrm{~J} / \mathrm{kg})$ is heat of formation of species $s$ and $f$ the number of degrees of freedom.

3.3b Mixture of perfect gases - Blottner model: In this model, the internal structure of the atoms and molecules are taken into account. Consequently, the specific heats and enthalpies are no longer constant and are dependent on the temperature. This is 
expressed as

$$
\begin{aligned}
C_{p, s} & =A_{1, s}(T), \\
h_{s} & =T A_{2, s}(T)+h_{s}^{0},
\end{aligned}
$$

where $A_{1, s}$ and $A_{2, s}$ are tabulated functions of temperature (Blottner et al 1971). In the present study, cubic spline interpolation is used to determine the intermediate values of $A_{1, s}$ and $A_{2, s}$.

3.3c Mixture of perfect gases - Liu-Vinokur model: As in the previous model, the internal structure of the atoms and molecules are taken into account. However, more exact methods utilizing the latest available spectroscopic constants are used in determining the thermodynamic properties (Liu \& Vinokur 1989). The properties are then curve fit using cubic splines and the spline coefficients tabulated as functions of temperature. This is expressed as

$$
\begin{aligned}
C_{p, s} & =C_{1, s}+C_{2, s}(z+T)+C_{3, s}\left(z^{2}+2 z T\right)+C_{4, s}\left(z^{3}+3 z^{2} T\right), \\
h_{s} & =T\left(C_{1, s}+C_{2, s} z+C_{3, s} z^{2}+C_{4, s} z^{3}\right)+h_{s}^{0},
\end{aligned}
$$

where $C_{1, s}, C_{2, s}, C_{3, s}$ and $C_{4, s}$ are tabulated values of the spline coefficients over a temperature range $250-25,000 \mathrm{~K}$ (in steps of $250 \mathrm{~K}$ ). The parameter $z$ is defined as

$$
z=T-\operatorname{int}(T / \Delta T) \Delta T
$$

In the above equation int $(\cdot)$ indicates the integer part of the argument.

For each of the gas models considered for the nonequilibrium case, the static pressure of the mixture is obtained from Dalton's law of partial pressures, i.e.,

$$
p=\rho \mathscr{R} T \sum_{s}\left(c_{s} / \mathscr{M}_{s}\right)=\rho \mathscr{R} T / \mathscr{M}
$$

The specific heat and enthalpy of the mixture are computed as weighted sums (the weights being the mass fractions of the species) of the species properties, i.e.,

$$
\begin{gathered}
C_{p_{f}}=\sum_{s} c_{s} C_{p, s}, \\
h=\sum_{s} c_{s} h_{s} .
\end{gathered}
$$

The frozen speed of sound $a_{f}$ for the nonequilibrium case is given by

$$
a_{f}=\left[\mathscr{R} C_{p_{f}} T /\left(\mathscr{M} C_{p_{f}}-\mathscr{R}\right)\right]^{1 / 2}
$$

The viscosity (Pa.s) of each individual species is obtained from curve fits due to Blottner et al (1971). These curve fits are of the form

$$
\mu_{s}=0.1 \exp \left[\left(A_{s} \ln T+B_{s}\right) \ln T+C_{s}\right]
$$

where $A_{s}, B_{s}$ and $C_{s}$ are constants. The thermal conductivity $(\mathrm{W} / \mathrm{m} \cdot \mathrm{K})$ of species $s$ is computed using a semi-empirical formula due to Eucken (1913)

$$
\kappa_{s}=\frac{\mu_{s} \mathscr{R}}{\mathscr{M}_{s}}\left(C_{p, s} \frac{\mathscr{M}_{s}}{\mathscr{R}}+\frac{5}{4}\right) .
$$

The transport properties of the gas mixture are then obtained through the use of mixing rules. The mixing rule due to Wilke (1950) is considered adequate for non-ionizing 
mixtures and is written for the coefficient of viscosity as

where

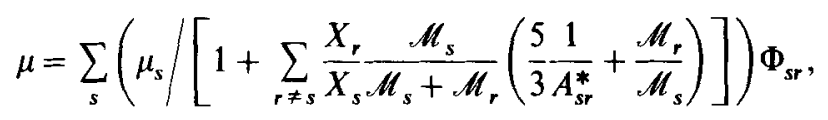

$$
\Phi_{s r}=\left[\frac{\mathscr{M}_{s}}{8\left(\mathscr{M}_{s}+\mathscr{M}_{r}\right)}\right]^{1 / 2}\left[F_{s r}+\left\{\frac{\mu_{s}}{\mu_{r}}\left(\frac{\mathscr{M}_{r}}{\mathscr{M}_{s}}\right)^{1 / 2}\right\}^{1 / 2} B_{s r}\right]^{2}
$$

The values of the constants $A_{s r}^{*}, B_{s r}$ and $F_{s r}$ are

$$
A_{s r}^{*}=\frac{5}{3}, \quad F_{s r}=1 \cdot 0, \quad B_{s r}=1 \cdot 0
$$

The same mixing rule is used for the coefficient of thermal conductivity with $\mu_{s}$ being replaced by $\kappa_{s}$ in (47).

In the present study, the mass diffusion is assumed to be binary. The justification for this is provided by the fact that air consisting of 5 species can be treated as a binary gas with the "heavy" particles $\mathrm{N}_{2}, \mathrm{O}_{2}$ and $\mathrm{NO}$ (nearly the same molecular weight) and "light" particles $\mathrm{N}$ and $\mathrm{O}$ (nearly the same atomic weight). The binary diffusion coefficient is obtained from the definition of the Lewis number which is assumed to be a constant.

$$
\mathrm{Le}=\rho C_{p} \mathscr{D} / \kappa \Rightarrow \mathscr{D}=\kappa \mathrm{Le} / \rho C_{p} .
$$

\subsection{Reaction model: Chemical nonequilibrium gas}

Consider a multicomponent system of $n$ species undergoing $m$ simultaneous elementary reactions. These reactions can be represented symbolically as

$$
\sum_{s=1}^{n} v_{l, s}^{\prime} A_{s} \rightleftharpoons \sum_{s=1}^{n} v_{l, s}^{\prime \prime} A_{s} \quad l=1,2, \ldots, m
$$

where $v_{l, s}^{\prime}, v_{l, s}^{\prime \prime}$ are stoichiometric coefficients and $A_{s}$ is chemical symbol of species $s$. Using the law of mass action, the rate of production of species $s$ is

$$
\dot{w}_{s}=\mathscr{M}_{s} \sum_{i=1}^{m}\left(v_{l, s}^{\prime \prime}-v_{l, s}^{\prime}\right)\left\{k_{f, l}(T) \prod_{r=1}^{n}\left[\rho \gamma_{r}\right]^{v_{l, r}^{\prime}}-k_{b, l}(T) \prod_{r=1}^{n}\left[\rho \gamma_{r}\right]^{v_{l, r}^{\prime \prime}}\right\}
$$

where $\gamma_{r}$ is the mole-mass ratio of species $r$ and $k_{f, l}$ and $k_{b, l}$ are, respectively, the forward and backward reaction rates of reaction $l$. These reaction rates are expressed in modified Arrhenius form (Blottner et al 1971) as:

$$
k_{f, l}=A_{f, l} T^{n_{f, l}} \exp \left(-E_{f, l} / k T\right) ; \quad k_{b, l}=A_{b, l} T^{n_{b, l}} \exp \left(-E_{b, l} / k T\right),
$$

where $A_{f, l}, A_{b, l}, n_{f, l}, n_{b, l}, E_{f, l}$ and $E_{b, l}$ are constants for a particular reaction $l$ and $k$ is the Boltzmann constant.

In the present work, the following reactions between the various species have been considered:

(1) $\mathrm{O}_{2}+\mathrm{M}_{1} \rightleftharpoons 2 \mathrm{O}+\mathrm{M}_{1}$

(2) $\mathrm{N}_{2}+\mathrm{M}_{2} \rightleftharpoons 2 \mathrm{~N}+\mathrm{M}_{2}$

(3) $\mathrm{NO}+\mathrm{M}_{3} \rightleftharpoons \mathrm{N}+\mathrm{O}+\mathrm{M}_{3}$

(4) $\mathrm{NO}+\mathrm{O} \rightleftharpoons \mathrm{O}_{2}+\mathrm{N}$

(5) $\mathrm{N}_{2}+\mathrm{O} \rightleftharpoons \mathrm{NO}+\mathrm{N}$ 


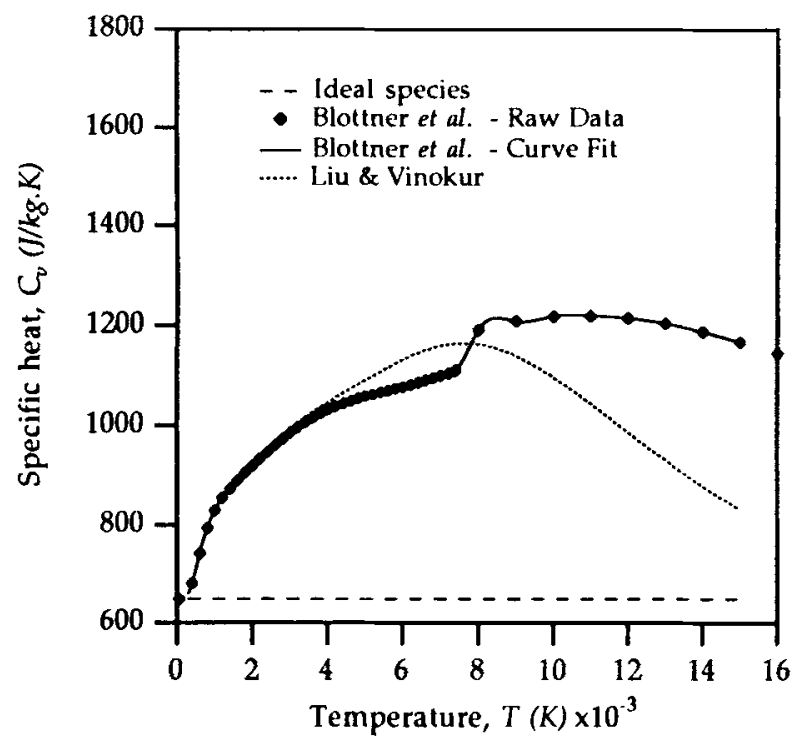

Figure 3. Temperature variation of the specific heat $\left(C_{1}\right)$ of molecular oxygen $\left(\mathrm{O}_{2}\right)$.

In reactions $1-3$ (also called three-body reactions), $M_{1}, M_{2}$ and $M_{3}$ represent the catalytic third body which undergoes no change during the reaction and could be any one of the species, $\mathrm{N}_{2}, \mathrm{O}_{2}, \mathrm{NO}, \mathrm{N}$, or O. Reactions 4 and 5 are called "shuffle" or exchange reactions. Therefore, there are 17 reactions in the above set.

\subsection{Discussion}

The inclusion of the internal structure of the species changes their thermodynamic properties. In figures 3-5, the specific heats (at constant volume) of $\mathrm{O}_{2}, \mathrm{NO}$, and $\mathrm{O}$ are

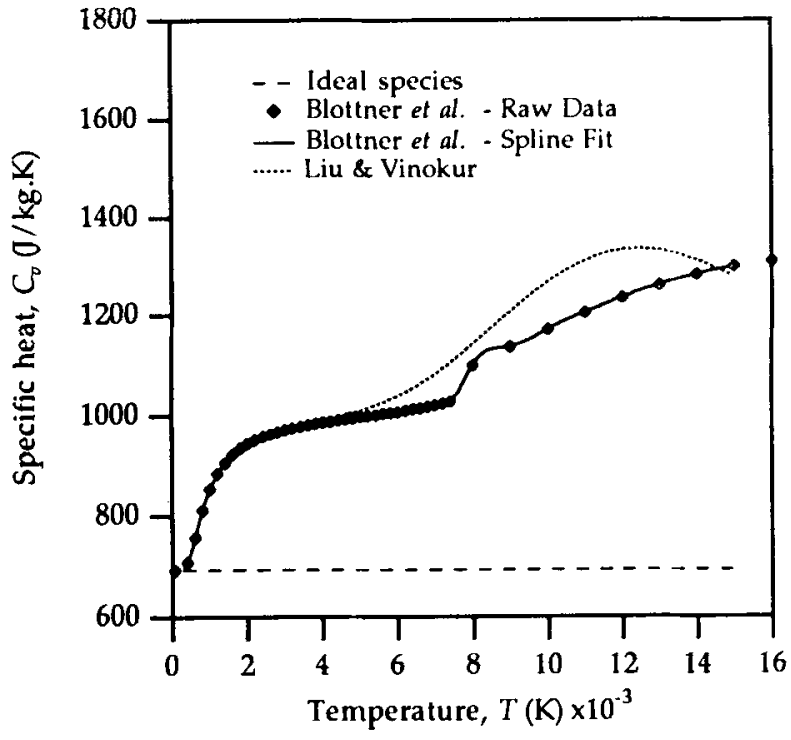

Figure 4. Temperature variation of the specific heat $\left(C_{v}\right)$ of nitric oxide (NO). 


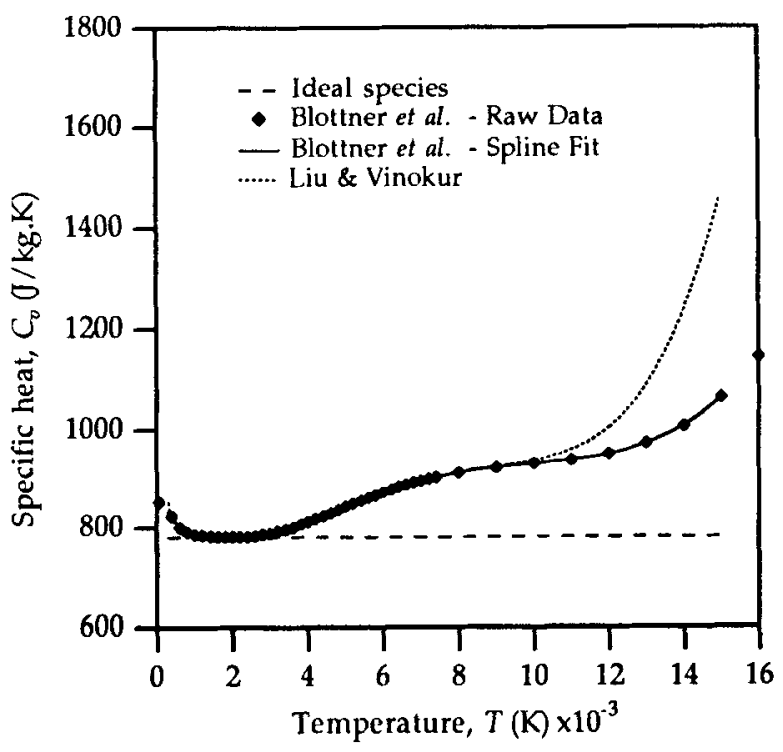

Figure 5. Temperature variation of the specific heat $\left(C_{v}\right)$ of atomic oxygen $(\mathrm{O})$.

plotted as functions of temperature. It is clearly seen that the species with no internal structure have a constant specific heat while those with internal structure have heat capacities that increase with increasing temperature (the heat capacity decreases in the diatomic species when their dissociation limits are reached). In the case of atomic oxygen, the agreement between the $C_{v}$ values of Blottner et al (1971) and those of Liu \& Vinokur (1989) is very good for temperatures less than about $8000 \mathrm{~K}$. Beyond this temperature, the differences are large. For the diatomic species, however, large differences occur between the models from around $3000 \mathrm{~K}$. The Liu-Vinokur model is to be considered as more accurate because it uses the latest available data for spectroscopic constants and intermolecular potentials in conjunction with accurate numerical methods.

\section{Analysis}

\subsection{Parabolization}

As mentioned earlier, the word "parabolized" in the PNS equations is a misnomer. The governing equations are actually hyperbolic-elliptic in the $\zeta$ direction. The ellipticity is introduced into the system due to the presence of the subsonic zone in the boundarylayer at the wall. This subsonic zone permits the pressure signals to travel upstream (upstream influence). Hence, a single-sweep space-marching method, wherein the spatial evolution (in $\xi$ ) of a line of initial data is computed, is not properly posed and leads to exponentially growing solutions called departure solutions. There are several ways of getting rid of this ellipticity (Vigneron et al 1978; Schiff \& Steger 1979). Following Vigneron et al (1978), an analysis was carried out for the various gas models considered in the present study. The details of the analyses are not given here but only the salient points are mentioned.

An eigenvalue (linear) analysis (Vigneron et al 1978; Prabhu \& Tannehill 1986; Prabhu et al 1988) of the governing equations reveals that the governing equations are 
hyperbolic-parabolic, i.e., well-posed for space marching in $\xi$ if and only if - (1) there is no flow separation in the axial $(\xi)$ direction, (2) the local Mach number is greater than unity in the inviscid part of the flowfield, and (3) only a fraction $\omega(0 \leqslant \omega \leqslant 1)$ of the streamwise pressure gradient is retained in the subsonic part of the flowfield. The magnitude of $\omega$ depends on the local streamwise Mach number and is given by the expression

$$
\omega=\min \left\{1, \sigma \bar{\gamma} \mathbf{M}_{\xi}^{2}\left[1+(\bar{\gamma}-1) \mathbf{M}_{\xi}^{2}\right]^{-1}\right\},
$$

where $\sigma(0 \leqslant \sigma \leqslant 1$, typically a value of 0.85 or less is used $)$ is a factor of safety to account for nonlinear effects neglected in the analysis. The parameter $\bar{\gamma}$ in the above equation is given by

$$
\bar{\gamma}= \begin{cases}\gamma & \text { (ideal gas) } \\ h / e & \text { (equilibrium air) } \\ \mathscr{M} C_{\boldsymbol{p}_{f}} /\left(\mathscr{M} C_{\boldsymbol{p}_{s}}-\mathscr{R}\right) & \text { (nonequilibrium air) }\end{cases}
$$

and the local streamwise Mach number is given by

$$
\mathbf{M}_{\xi}=\hat{U} /(a|\nabla \xi|)
$$

where the speed of sound $a$ is given by (21) for an ideal gas, by the second correlation of (25) for equilibrium air, or by (44) for chemical nonequilibrium air.

Note that when $\omega=0$ the streamwise pressure gradient is completely omitted and when $\omega=1$ the streamwise pressure gradient is completely retained. In the inviscid part of the flowfield, since the Mach number must be greater than unity, the streamwise pressure gradient is completely retained. In the subsonic viscous region close to the wall only a fraction of $\partial p / \partial \xi$ is retained.

\subsection{Treatment of the streamwise pressure gradient}

From the preceding paragraphs, it is clear that for a single sweep space-marching solution to be viable, the streamwise pressure gradient has to be treated specially. The streamwise inviscid flux $\mathbf{E}^{i}$ is now split into two parts $\mathbf{E}^{i^{*}}$ and $\mathbf{P}^{i}$ as shown below

$$
\mathbf{E}^{i}=\mathbf{E}^{i^{*}}+\mathbf{P}^{i}
$$

where $\mathbf{E}^{i^{*}}$ and $\mathbf{P}^{i}$ are

$$
\begin{aligned}
\mathbf{E}^{i^{*}} & =(1 / J)\left\{\rho \hat{U}, \rho u \hat{U}+\xi_{x} \omega p, \rho w \hat{U}+\xi_{z} \omega p,\left(\rho e_{t}+p\right) \hat{U}\right\}^{T}, \\
\mathbf{P}^{i} & =(1 / J)\left\{0, \xi_{x}(1-\omega) p, \xi_{z}(1-\omega) p, 0\right\}^{T},
\end{aligned}
$$

for the ideal/equilibrium case, and

$$
\mathbf{E}^{i^{*}}=\frac{1}{J}\left(\begin{array}{c}
\rho \hat{U} \\
\rho u \hat{U}+\xi_{x} \omega p \\
\rho w \hat{U}+\xi_{z} \omega p \\
\rho H \hat{U} \\
\rho c_{1} \hat{U} \\
\rho c_{2} \hat{U} \\
\vdots \\
\rho c_{n-1} \hat{U}
\end{array}\right), \quad \mathbf{P}^{i}=\frac{1}{J}\left(\begin{array}{c}
0 \\
\xi_{x}(1-\omega) p \\
\xi_{z}(1-\omega) p \\
0 \\
0 \\
0 \\
\vdots \\
0
\end{array}\right)
$$

for the nonequilibrium case. 
The governing equations (1) or (8) can now be written as

$$
\frac{\partial}{\partial \xi}\left(\mathbf{E}^{i^{*}}\right)+\frac{\partial}{\partial \zeta}\left(\mathbf{G}^{i}-\mathbf{G}^{v}\right)=j\left(\mathbf{S}^{i}-\mathbf{S}^{v}\right)+\left[\mathbf{W}^{c}\right]-\frac{\partial}{\partial \xi}\left(\mathbf{P}^{i}\right) .
$$

Normally, the last term of (61) is not included in the computations and the systems of equations that is actually solved is

$$
\frac{\partial}{\partial \xi}\left(\mathbf{E}^{i^{*}}\right)+\frac{\partial}{\partial \zeta}\left(\mathbf{G}^{i}-\mathbf{G}^{v}\right)=j\left(\mathbf{S}^{i}-\mathbf{S}^{v}\right)+\left[\mathbf{W}^{c}\right]
$$

\section{Numerical method}

The PNS equations, (62), cannot be solved analytically and must be solved numerically. In the present study, a finite-difference approach has been taken for the numerical solution of the governing equations. The method of discretisation of the solution domain, the numerical algorithm, and the numerical implementation of boundary conditions are discussed in this section.

\subsection{Discretisation and grid generation}

As mentioned earlier, the computational domain consists of two boundaries - (1) an outer freestream boundary and, (2) an inner wall boundary. The outer boundary is chosen in such a way that the bow shock lies completely within this domain. The domain is then discretised algebraically for implementation of a finite-difference procedure. In this procedure, at any given $\xi$ station, a point on the body surface and a point on the outer boundary are connected by a straight line and grid points distributed on this line using the following stretching function

$$
\begin{aligned}
& s(\zeta)=1-\beta\left[\frac{(\beta+1)^{1-\zeta}-(\beta-1)^{1-\zeta}}{(\beta+1)^{1-\zeta}+(\beta-1)^{1-\zeta}}\right], \\
& \zeta=(k-1) /(N K-1), \quad k=1,2, \ldots, N K,
\end{aligned}
$$

where $\beta(\beta>1)$ is the stretching function and $N K$ the total number of points on the grid line. Note that $s(0)=0$ and $s(1)=1$ and the points are clustered close to the wall for values of $\beta$ close to 1 . Such clustering is necessary for good resolution of the subsonic viscous layer.

The coordinates of the grid points on the grid line are obtained from

$$
\begin{aligned}
& x(\xi, \zeta)=x_{w}(\xi)+n_{1}(\xi) s(\zeta) \delta(\xi), \\
& y(\xi, \zeta)=y_{w}(\xi)+n_{2}(\xi) s(\zeta) \delta(\xi),
\end{aligned}
$$

where $n_{1}$ and $n_{2}$ are direction cosines of the unit vector along the grid line and $\delta$ is the linear distance of the outer boundary from the body surface.

\subsection{Algorithm}

An implicit, noniterative finite-difference scheme is used to solve the PNS equations, (62). This algorithm is an adaptation of one developed by Tannehill et al (1982). 
Associating the index $i$ with the streamwise direction $\zeta$ and the index $k$ with the normal direction $\zeta$, this algorithm in delta form is

$$
\begin{aligned}
\left\{\mathbf{A}_{i, k}^{*}\right. & \left.+\Delta \xi \frac{\partial}{\partial \zeta}\left(\mathbf{B}_{i, k}-\mathbf{M}_{i, k}\right)-\mathbf{D}_{2}\right\} \Delta \mathbf{Q}_{i, k}= \\
& -\Delta \xi\left\{\frac{\partial}{\partial \zeta}\left(\mathbf{G}_{i, k}^{i}-\mathbf{G}_{i, k}^{v}\right)+\mathbf{S}_{i, k}^{i}-\mathbf{S}_{i, k}^{v}-\left[\mathbf{W}_{i, k}^{c}\right]\right\}+\mathbf{D}_{4} \mathbf{E}^{i^{*}},
\end{aligned}
$$

where the change in the vector of dependent variables is

$$
\Delta \mathbf{Q}_{i, k}=\mathbf{Q}_{i+1, k}-\mathbf{Q}_{i, k},
$$

and the Jacobian matrices of the various fluxes are,

$$
\mathbf{A}^{*}=\frac{\partial \mathbf{E}^{i *}}{\partial \mathbf{Q}}, \quad \mathbf{B}=\frac{\partial \mathbf{G}^{i}}{\partial \mathbf{Q}}, \quad \mathbf{M}=\frac{\partial \mathbf{F}^{v}}{\partial \mathbf{Q}} .
$$

The derivative $\partial / \partial \zeta$ is replaced by the conventional 3-point central-difference operator. $\mathbf{D}_{2}$ and $\mathbf{D}_{4}$ are second-order implicit and fourth-order explicit smoothing operators, respectively. These are required to stabilize the central-difference algorithm. In the linearization of the viscous flux, the transport properties are assumed to be locally constant. Note that all the source terms have been lagged in the present formulation.

The left hand side of the algorithm, (67), corresponds to a block-tridiagonal system of equations. The blocks are square matrices of order 4 and order $(n+3)$ for the ideal/equilibrium gas case and the nonequilibrium case, respectively. Specifically, for the five-species nonequilibrium air model considered in the present calculations, these blocks are square matrices of order 8 .

The code developed in the present study is a unified code for all the three gas models. Therefore, the block tridiagonal solver developed here is meant for systems of any block order $m(m>1)$. The penalty paid in using a general solver instead of using one for order 4 and one for order 8 is not substantially large.

\subsection{Decoding the $\mathbf{Q}$ vector}

At the end of the solution step given by (68), we have just the vector $\mathbf{Q}_{i+1, k}$. From this vector we have to extract all the thermodynamic and transport coefficients and apply the boundary conditions. The decoding procedure and implementation of the boundary conditions for each of the gas models are considered in what follows.

5.3a Ideal air: Decoding the $\mathbf{Q}$ vector for an ideal gas is rather simple. One can easily obtain the variables $\rho, u, w$, and $e_{t}$ from $\mathbf{Q}$. Knowing the variables $u, w$, and $e_{t}$, the variable $e$ is obtained from (3). The variables $T, p, a, \mu$ and $\kappa$ follow from (19)-(23), respectively.

5.3b Equilibrium air: Decoding the $\mathbf{Q}$ vector for this case is a little more involved. As before, we can easily obtain the variables $\rho, u, w$ and $e_{t}$ from $\mathbf{Q}$. Again, using (3) and the values of $u, w$ and $e_{t}$ we can compute the value of $e$. Knowing $e$ and $\rho$, we can use the correlations given in (25) to determine the values of $p, a$ and $T$. Using (27) and (28), we 
compute the parameters $\bar{\gamma}$ and $\bar{\beta}$. The transport properties are easily determined from (32).

5.3c Nonequilibrium air: Decoding the $\mathbf{Q}$ vector for this case is again quite involved. We can easily obtain the variables $\rho, u, w, H$, and $c_{1}, c_{2}, \ldots, c_{n-1}$ from $\mathbf{Q}$. The mass fraction $c_{n}$ is computed from (11). Using (10) and the values of $u, w$, and $H$ we can compute the value of $h$. Knowing $h$ and the mass fractions $c_{1}, c_{2}, \ldots, c_{n}$, we compute the temperature $T$ of the mixture iteratively using the Newton-Raphson method.

$$
T^{p+1}=T^{p}-\frac{\sum_{s=1}^{n} c_{s} h_{s}\left(T^{p}\right)-h}{\sum_{s=1}^{n} c_{s} C_{p s}\left(T^{p}\right)},
$$

where $p$ is an iteration index. The Newton-Raphson method usually requires no more than 3 to 5 iterations for convergence. Once the temperature has been computed, the other thermodynamic and transport properties (for both the species and the mixture) are computed using expressions listed in the section on gas models.

\subsection{Numerical boundary conditions}

The implementation of boundary conditions (discussed in the section on governing equations) is considered here. In the implicit portion of the algorithm (left hand side of 67) boundary conditions are applied to only first-order accuracy. This is done to preserve the block tridiagonal structure of the matrix. After the integration step, the boundary conditions are updated explicitly. This is discussed for each of the gas models in what follows.

5.4a Ideal gas: At the wall, the pressure is assumed to be the same as the pressure one grid point above it (17). Using this value of pressure and the given wall temperature $T_{w}$, the value of the mass density of the wall can be computed. No-slip conditions are implemented by setting the values of $u$ and $w$ to zero.

At the freestream boundary, $\mathbf{Q}$ at the grid point is updated using the values at the grid point just in the interior of the domain, i.e., a zero-gradient condition is imposed on $\mathbf{Q}$.

5.4b Equilibrium air: At the wall, the pressure is assumed to be the same as the pressure one grid point above it (17). Now the temperature of the wall is specified as $T_{w}$. Using the second correlation of (26), i.e.,

$$
T_{w}=T_{w}\left(p_{w}, \rho_{w}\right)
$$

the wall density $\rho_{w}$ is determined iteratively using a secant method (Prabhu \& Tannehill 1986). Using the first correlation of (26), the enthalpy at the wall is determined for given values of $p$ and $\rho$. The internal energy at the wall is then updated using the definition of the enthalpy

$$
e_{w}=h_{w}-\left(p_{w} / \rho_{w}\right)
$$

This is done in order to ensure consistency in the use of curve fits. No-slip conditions are implemented by setting the values of $u$ and $w$ to zero.

At the freestream boundary, a zero-gradient condition is imposed on $\mathbf{Q}$. 
5.4c Nonequilibrium air: At the wall, the pressure is assumed to be the same as the pressure one grid point above it (17). Now the temperature of the wall is specified as $T_{w}$. The mass fractions of the species are computed using zeroth-order extrapolation from the interior. Using these values of mass fractions, temperature, and pressure, the density of the gas mixture is computed from the equation of state (41). No-slip conditions are implemented by setting the values of $u$ and $w$ to zero.

At the freestream boundary, a zero-gradient condition is imposed on $\mathbf{Q}$.

\section{Results}

\subsection{Test conditions}

The laminar hypersonic flow past a sharp right circular cone has been computed in the present study. The details of the test conditions are given in table 1. The ambient conditions correspond to an altitude of $61 \mathrm{~km}$ approximately. The Mach and Reynolds numbers for these conditions are 25 and $0.13 \times 10^{6}$ per $\mathrm{m}$, respectively.

\subsection{Preliminaries}

The calculations were started at $x=1 \times 10^{-3} \mathrm{~m}$ from the tip of the cone. This was done to avoid the singularity at $x=0$ where $z=0$. The line of initial data was initialised using freestream values and this line was marched downstream to a location of $x=1.276 \mathrm{~m}$ with the marching step-size varying linearly from $\Delta x=5 \times 10^{-4} \mathrm{~m}$ to $\Delta x=1.2 \times 10^{-3} \mathrm{~m}$ over 1500 steps. The spacing at the wall was also varied linearly from $\Delta z_{b}=5 \times 10^{-5} \mathrm{~m}$ to $\Delta z_{b}=10^{-4} \mathrm{~m}$. The outer boundary height, however, was kept fixed at $\delta(x)=0.5 \mathrm{~m}$. The number of grid points was chosen after detailed grid sensitivity studies were carried out. A sampling of the results obtained from the various studies in this work is presented in what follows (some more results and details can be found in Prabhu 1993).

In the presentation of the results, the following nondimensional quantities - surface pressure coefficient $C_{p}$, skin-friction coefficient $C_{f}$, and Stanton number $S t$ have been defined as

$$
C_{p_{x}}=\frac{p_{w}-p_{x}}{\frac{1}{2} \rho_{x} U_{x}^{2}}, \quad C_{f}=\frac{\tau_{w}}{\frac{1}{2} \rho_{s} U_{x}^{2}}, \quad \mathrm{St}=\frac{-\dot{q}_{2}}{\rho_{x} U_{x}\left(H_{x}-H_{w}\right)},
$$

where $p_{w}$ is wall pressure (Pa), $\tau_{w}$ is surface shear stress $\left(\mathrm{N} / \mathrm{m}^{2}\right), \dot{q}_{w}\left(\mathrm{~W} / \mathrm{m}^{2}\right)$ and $H_{w}(\mathrm{~J} / \mathrm{kg})$ are respectively, the total heating and the total enthalpy at the wall. The total heating at

Table 1. Test conditions.

\begin{tabular}{ll}
\hline Cone half angle $\left(\theta_{c}\right)$ & 10 \\
Freestream velocity $\left(U_{x}\right)$ & $8100 \mathrm{~m} / \mathrm{s}$ \\
Freestream pressure $\left(p_{x}\right)$ & $20.35 \mathrm{~Pa}$ \\
Freestream temperature $\left(T_{x_{x}}\right)$ & $252.6 \mathrm{~K}$ \\
Wall temperature $\left(T_{w}\right)$ & $1200 \mathrm{~K}$ \\
Freestream $\mathrm{N}_{2}$ mass fraction $\left(c_{\mathrm{N}}\right)$ & 0.79 \\
Freestream $\mathrm{O}_{2}$ mass fraction $\left(c_{\mathrm{O}_{2}}\right)$ & 0.21 \\
Noncatalytic wall & \\
\hline
\end{tabular}


the wall is conductive heating in the case of an ideal gas, and the sum of conductive and diffusive heating in the case of equilibrium and nonequilibrium air.

\subsection{Grid study}

The sensitivity of the computed solution to varying grid sizes was studied first to establish the consistency of the numerical scheme. Six different grid sizes $-N K=67$, $75,101,125,151$, and 167 were tried for the ideal gas $(\gamma=1 \cdot 4)$ and the nonequilibrium air models. Some of the results for the nonequilibrium case are presented here and the grid sensitivity of the solution for the ideal gas case can be found in Prabhu (1993).

The axial variation of the surface pressure coefficient $C_{p_{w}}$ for the various grid sizes is shown in figure 6 . The impact of the grid resolution is clearly seen in the picture. As the grid is refined the solution changes. Though the changes are very pronounced for the coarser grids, for the two finest grids ( $N K=151$ and 167) the differences between the solutions are negligibly small. This indicates the independence (from the grid) of the solution. The Newtonian value of the surface pressure coefficient is also shown in the figure. The deviation between the PNS results and the Newtonian results is due to the viscous-inviscid interaction which is rather strong at the tip of the cone for the chosen Mach number and Reynolds number in the present study.

Figure 7 shows the pressure profiles at the base of the cone (at $x=1.276 \mathrm{~m}$ ) for various grid sizes. The grid size has a significant impact on the pressure. The shock gets progressively sharper and the post-shock pressure rises with grid fineness. For the finest two grids, however, the differences in the profiles are negligibly small.

The pressure profiles also show oscillations which are characteristic of centraldifference schemes. The amplitudes of these oscillations grow with the grid fineness. Recall that central differencing has been used for the inviscid fluxes. This means that the flux differencing is not biased in the direction of signal propagation and hence, the numerical damping that is necessary for algorithm stability will smear any discontinuities

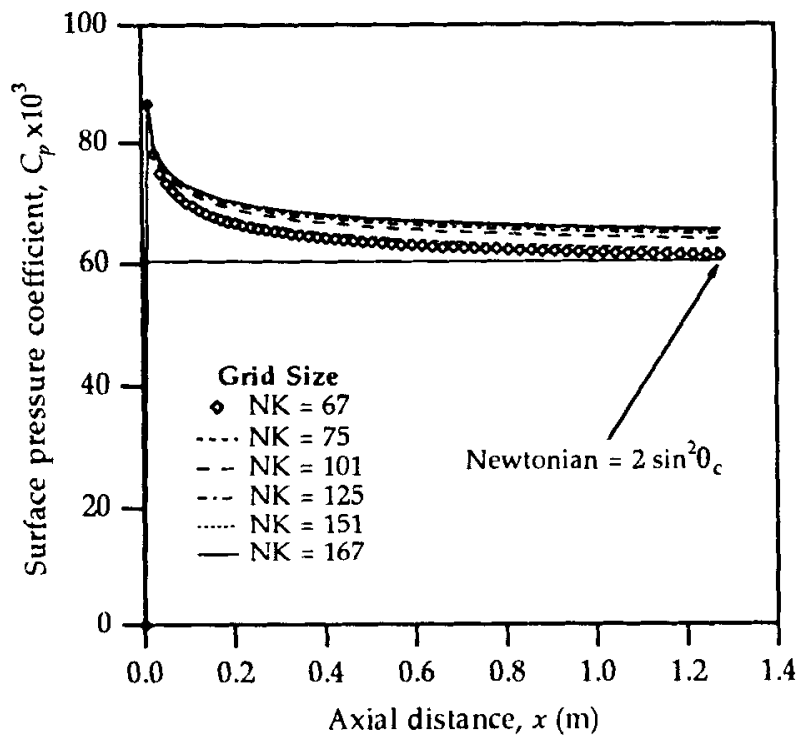

Figure 6. Axial variation of the computed surface pressure coefficient for various grid sizes for the nonequilibrium gas model of Blottner et al (1971). The Newtonian pressure coefficient distribution is also shown. 


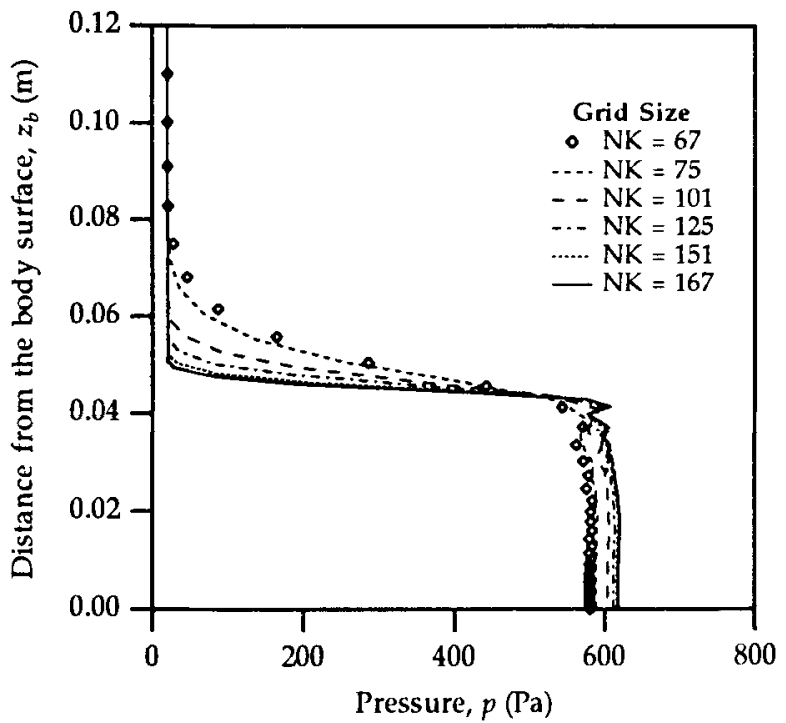

Figure 7. Effect of grid size on the static pressure. Profiles of $p$ at $x=1.276 \mathrm{~m}$ for various grid sizes. The oscillations are due to the use of central differences for the inviscid fluxes.

that are captured as part of the solution. In the coarse mesh the captured outer shock smears over quite a few grid points which are spaced rather widely apart. In the fine mesh the shock still spreads over nearly the same number of grid points which are, however, quite closely spaced resulting in a sharper definition of the captured shock. It must be mentioned here that the numerical smoothing coefficient was kept constant (and at a low value) across all grid sizes. This causes a problem in the finer grids where high-frequency errors need to be damped out with a larger amount of artificial viscosity. This explains the increased amplitude of the oscillations for the finest grids.

Figure 8 shows the temperature profiles at the base of the cone (at $x=1.276 \mathrm{~m}$ ) for various grid sizes. The agreement is good at all points except at the points in the shock

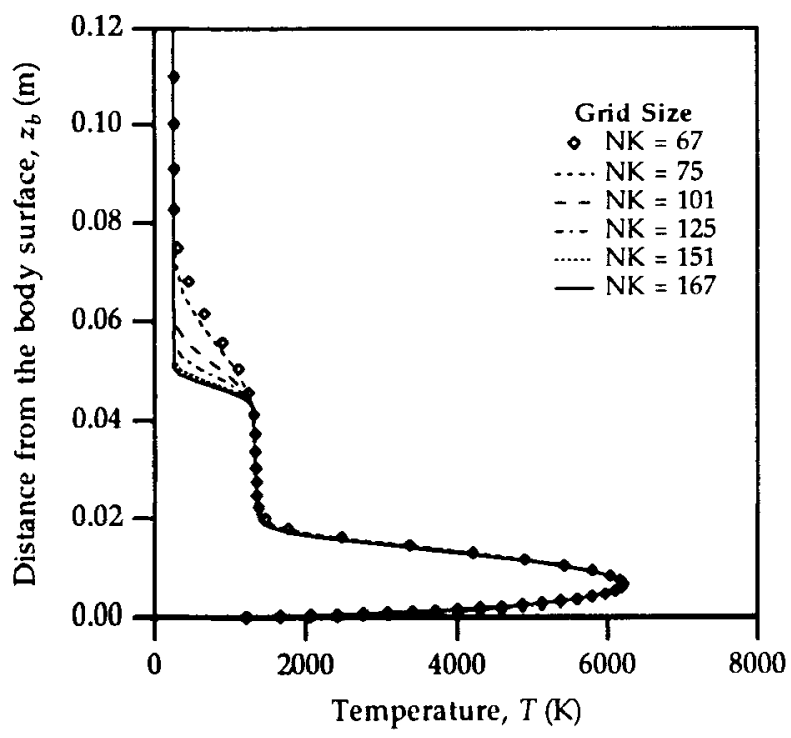

Figure 8. Effect of grid size on the static temperature. Profiles of $T$ at $x=1.276 \mathrm{~m}$ for various grid sizes. 


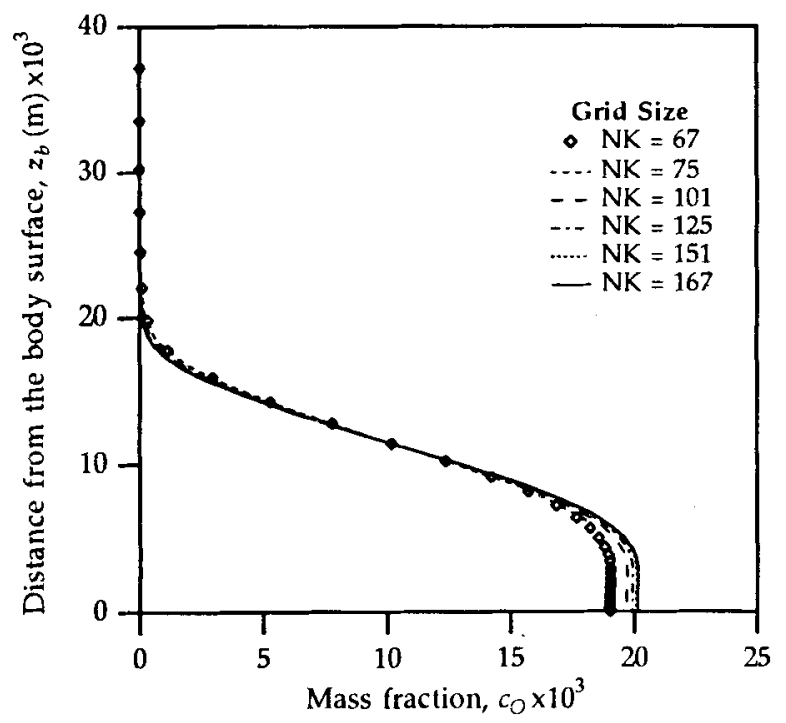

Figure 9. Effect of grid size on the mass fraction of $\mathrm{O}$ (atomic oxygen). Profiles of $c_{0}$ at $x=1.276 \mathrm{~m}$ for various grid sizes.

region. The grid fineness has little or no impact on the peak temperature in the boundary layer and the temperature profiles do not exhibit the oscillations seen in the pressure.

The mass fraction profiles of two representative species - atomic oxygen $(\mathrm{O})$ and molecular nitric oxide (NO) are shown in figures 9 and 10, respectively. Again, the grid refinement has a significant influence on the chemistry. The changes in the mass fractions, however, decrease at the finest grid levels. Large changes in the chemistry in the coarser grids can pose problems in radiation calculations which are very sensitive to changes in the species concentrations.

On the basis of the grid sensitivity study for both the ideal gas and nonequilibrium air gas models, a grid size of $N K=151$ points was chosen for all subsequent calculations.

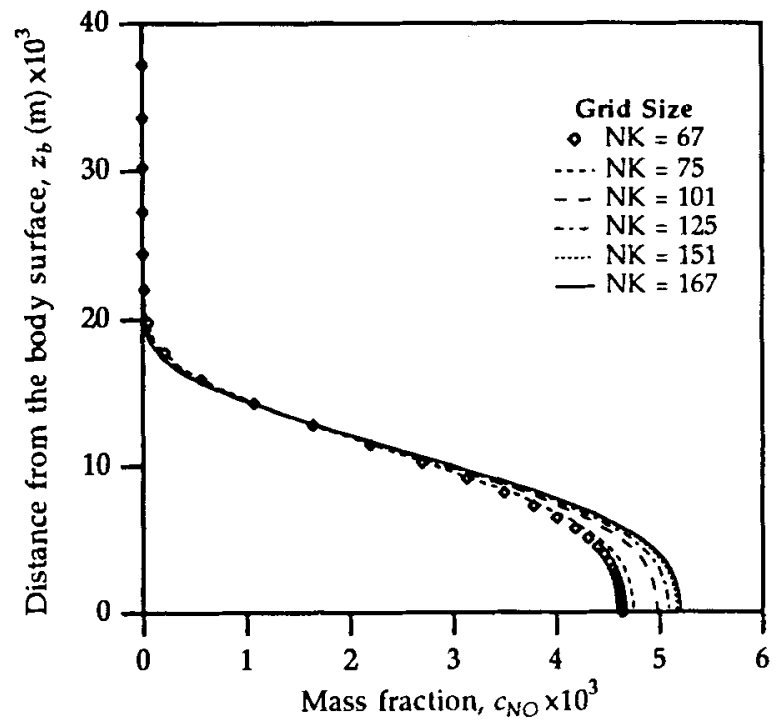

Figure 10. Effect of grid size on the mass fraction of NO (nitric oxide). Profiles of $c_{\mathrm{NO}}$ at $x=1.276 \mathrm{~m}$ for various grid sizes. 
This was done in order to avail the gain in accuracy (with finer grids) while keeping the computing times down to a reasonable level.

\subsection{Species structure study}

After having studied the grid dependence of the computed solution for the nonequilibrium air case, the impact of the thermodynamic properties of the constituent species was studied for three models of the internal structure - (1) ideal species model, (2) model due to Blottner et al (1971), and (3) model due to Liu \& Vinokur (1989).

The pressure profiles at $x=1.276 \mathrm{~m}$ for the three species structure models are shown in figure 11. While the results of the Blottner and Liu-Vinokur models are indistinguishable, they are very different from those of the ideal species model, especially in the shock region. At the body, however, all three models predict nearly the same surface pressure. This is understandable because the pressure being a "mechanical" variable is less dependent on the internal structure of the species. The shock standoff distance, on the other hand, depends on the thermodynamic properties of the species. In the region behind the shock where there are very high temperatures, the internal degrees of freedom of the species are excited.

The temperature profiles at $x=1.276 \mathrm{~m}$ for the three species structure models are shown in figure 12. Again, the results of the Blottner and Liu-Vinokur models are indistinguishable. However, these results are very different from those of the ideal species model. The post-shock and peak boundary layer temperatures are higher for the ideal species model. This is purely due to the internal structure of the species. The internal structure enables the species to absorb the collisional energy thereby lowering the temperatures.

Since the ideal species lack internal structure, the collisional energy can only lead to increased dissociation. This is clearly seen in figures 13 and 14 which depict the mass fraction profiles of atomic oxygen $(\mathrm{O})$ and nitric oxide $(\mathrm{NO})$, respectively. While the

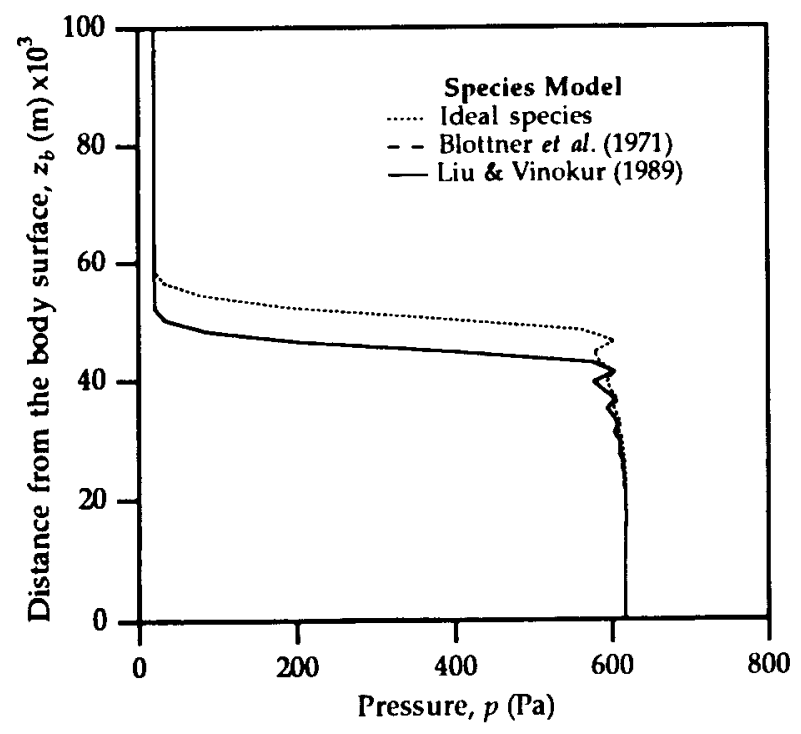

Figure 11. Effect of the internal structure of atoms and molecules on the static pressure. Pressure profiles at $x=1.276 \mathrm{~m}$ are shown here for the non equilibrium gas model. 


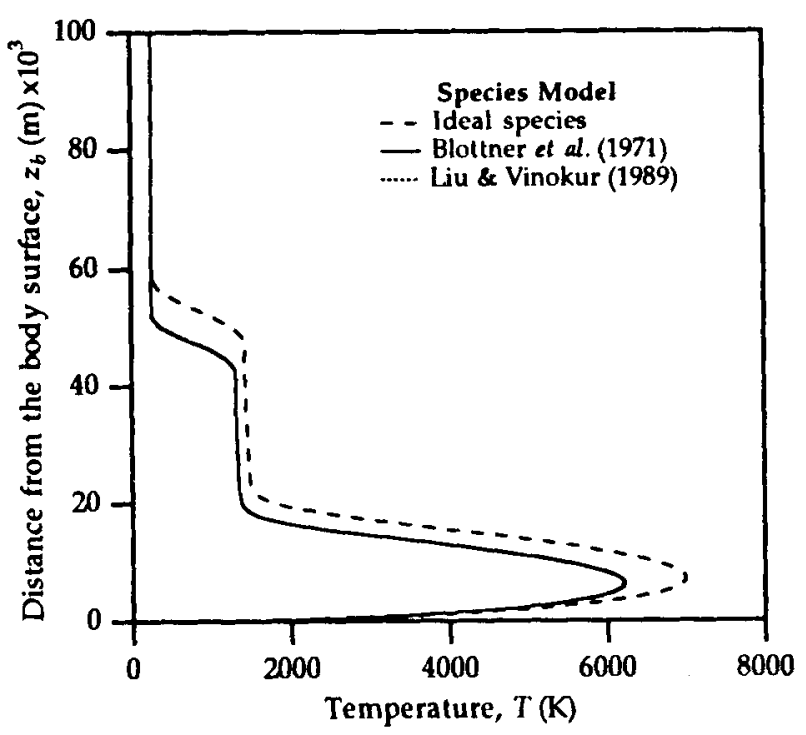

Figure 12. Effect of the internal structure of atoms and molecules on the temperature. Temperature profiles at $x=$ $1.276 \mathrm{~m}$ are shown here for the nonequilibrium model.

difference between the results of the Blottner and Liu-Vinokur models are negligibly small, the ideal species model leads to larger amount of dissociation.

\subsection{Gas model study}

The principal aim of this portion of the work is to study the ideal, equilibrium, nonequilibrium (Liu--Vinokur) air models in the context of external hypersonic flows and determine their influence on the thermodynamic environment. Again a fine grid ( $N K=151$ ) was chosen and identical marching step sizes and grid spacings were used for all the computations.

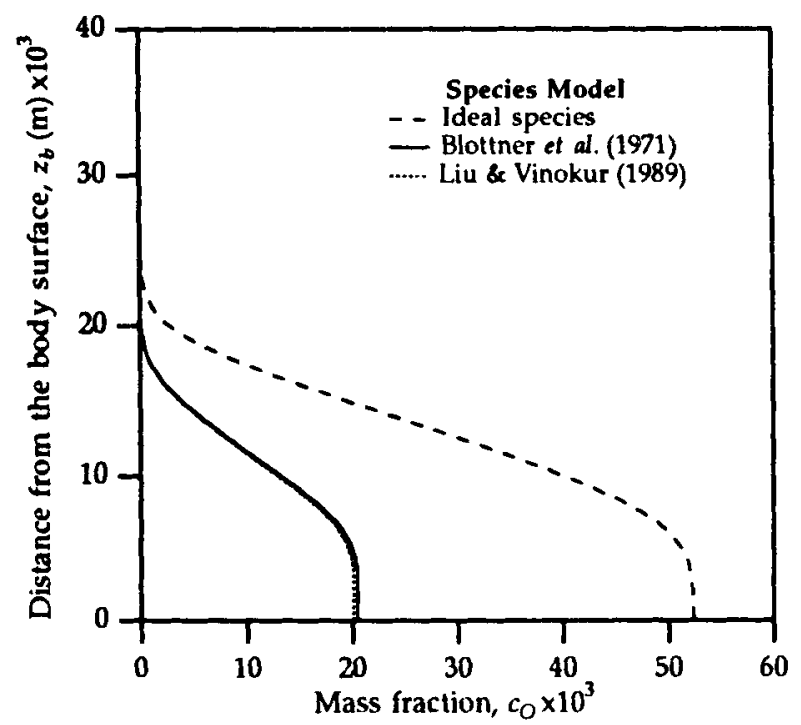

Figure 13. Effect of the internal structure of atoms and molecules on the chemistry. Mass fraction profiles of atomic oxygen (O) at $x=1.276 \mathrm{~m}$ are shown here for the nonequilibrium model. 


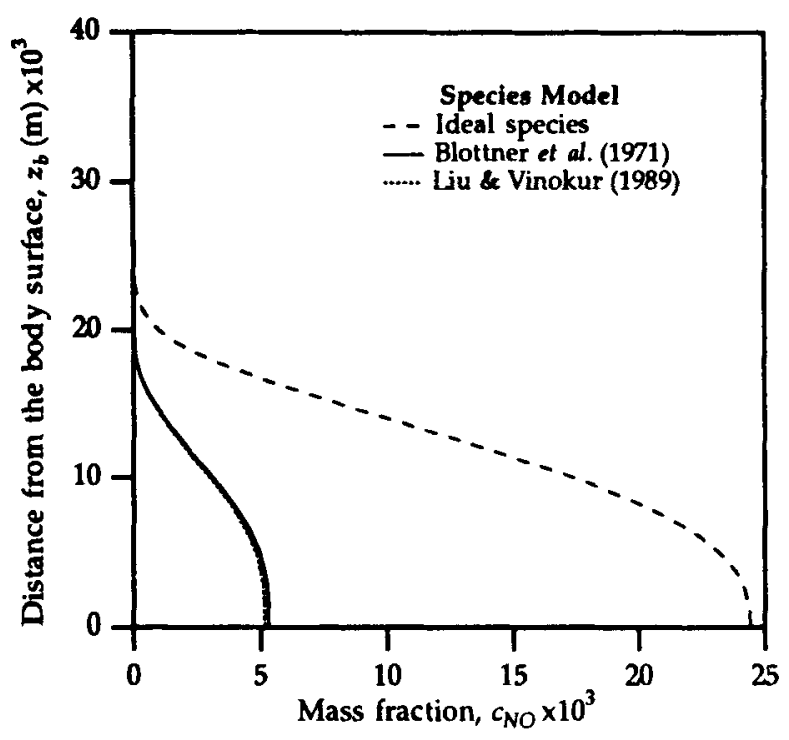

Figure 14. Effect of the internal structure of atoms and molecules on the chemistry. Mass fraction profiles of nitric oxide (NO) at $x=1.276 \mathrm{~m}$ are shown here for the nonequilibrium model.

The pressure and temperature profiles at $x=1.276 \mathrm{~m}$ are shown in figures 15 and 16 , respectively. There are differences in the shock standoff distances for the three gas models with the shock standoff distance being the largest for ideal gas and the smallest for equilibrium air. The elevated temperatures behind the shock serve to excite the internal degrees of freedom of the gases that constitute the mixture. The effect of this is to alter the value of $\bar{\gamma}$ behind the shock, thus altering the standoff distance. The peak temperature within the boundary layer are significantly different between the three models. The effect of chemical reactions (finite or infinite rates) is to drain the kinetic energy contained in the fluid and use it in the chemical reactions and/or excitation of

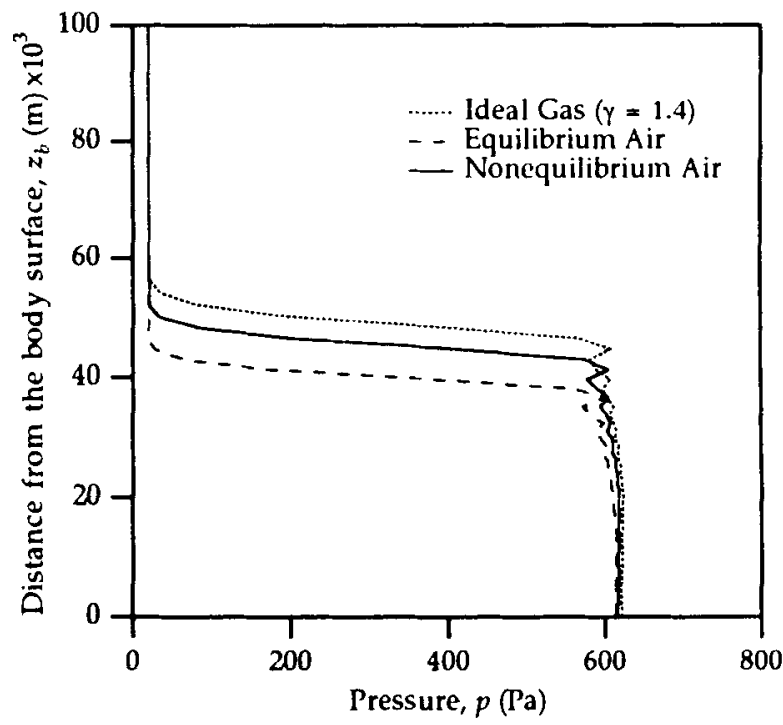

Figure 15. Pressure profiles at $x=1.276 \mathrm{~m}$ for ideal, equilibrium and nonequilibrium air models. Oscillations in the pressure are typical of centraldifference schemes. 


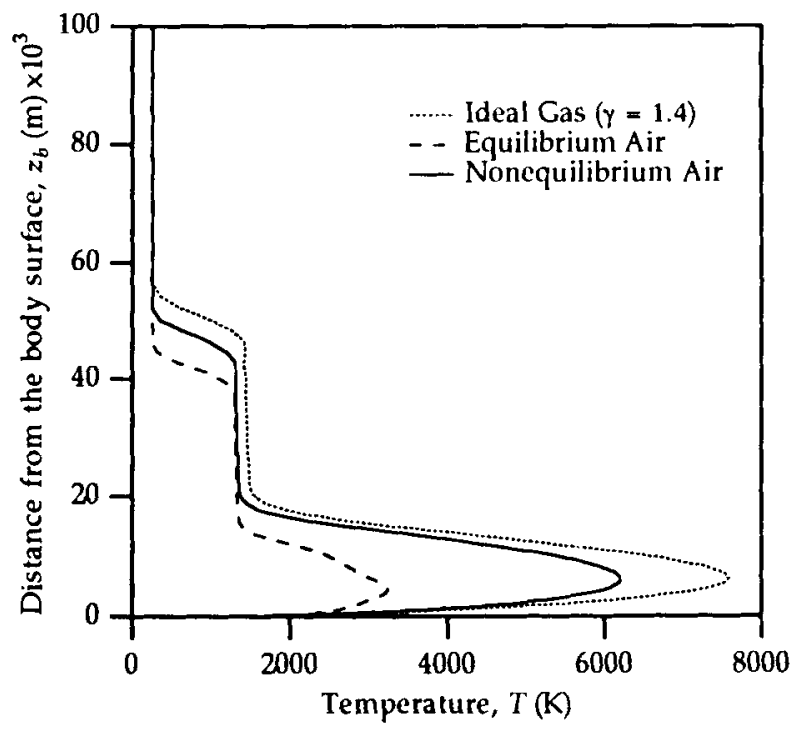

Figure 16. Temperature profiles at $x=1.276 \mathrm{~m}$ for ideal, equilibrium and nonequilibrium air models.

internal states of the constituent species. This shows up as an overall reduction in the peak temperature in the boundary layer.

The departure of the gas from ideal behaviour can be studied using the two parameters $\bar{\gamma}$ and $\bar{\beta}$ defined previously. The profiles of $\bar{\gamma}$ and $\bar{\beta}$ at $x=1.276 \mathrm{~m}$ are depicted in figures 17 and 18 , respectively. Note that $\bar{\gamma}$ changes from its usual value of 1.4 immediately before the shock to about 1.3 for the nonequilibrium case and about $1.18 \mathrm{~m}$ in the equilibrium case. This is to be expected because the internal energy and enthalpy of the species are dependent on the local temperature which varies depending

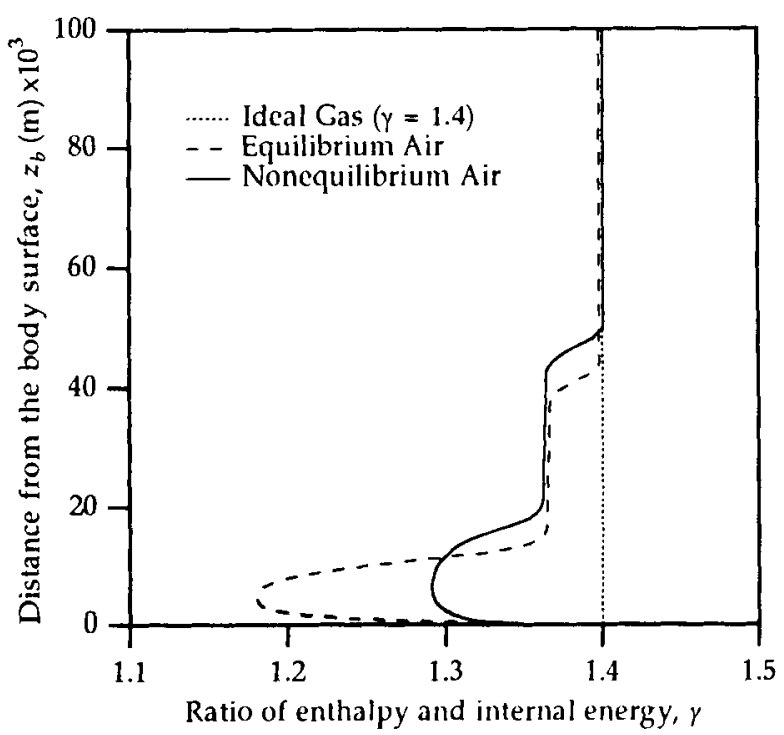

Figure 17. Profiles of the ratio of enthalpy and internal energy at $x=1.276 \mathrm{~m}$ for ideal, equilibrium and nonequilibrium air models. 


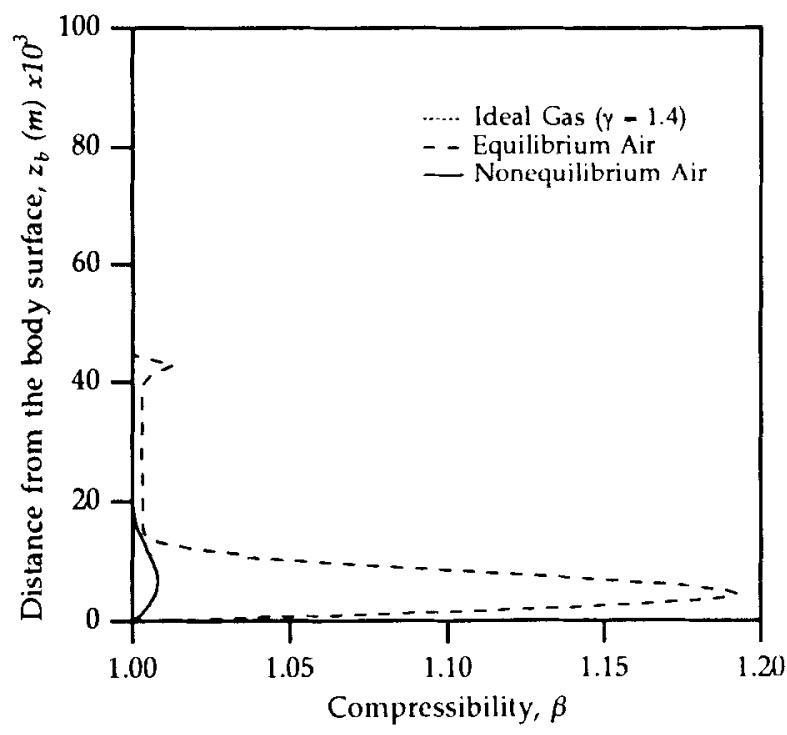

Figure 18. Profiles of the gas compressibility $(\beta)$ at $x=1.276 \mathrm{~m}$ for ideal, equilibrium and nonequilibrium air models.

on the gas model. The parameter $\bar{\beta}$ which is a ratio of the local molecular weight to the average molecular weight varies from 1 to about 1.2. This parameter becomes important only in the reaction zone where the mass concentrations vary. Hence, there is no difference at the shock but there is substantial variation in the boundary layer. For the equilibrium air case, $\bar{\beta}$, varies at the shock. This difference can perhaps be attributed to the piecewise continuity of the curve fits for equilibrium air. This effect has to be studied in greater detail.

The axial variation of the surface pressure coefficient $\left(C_{p}\right)$ is shown in figure 19 . The ideal and nonequilibrium surface pressures are very nearly the same but differ from that for equilibrium air. This is quite surprising since the surface pressure is expected to be

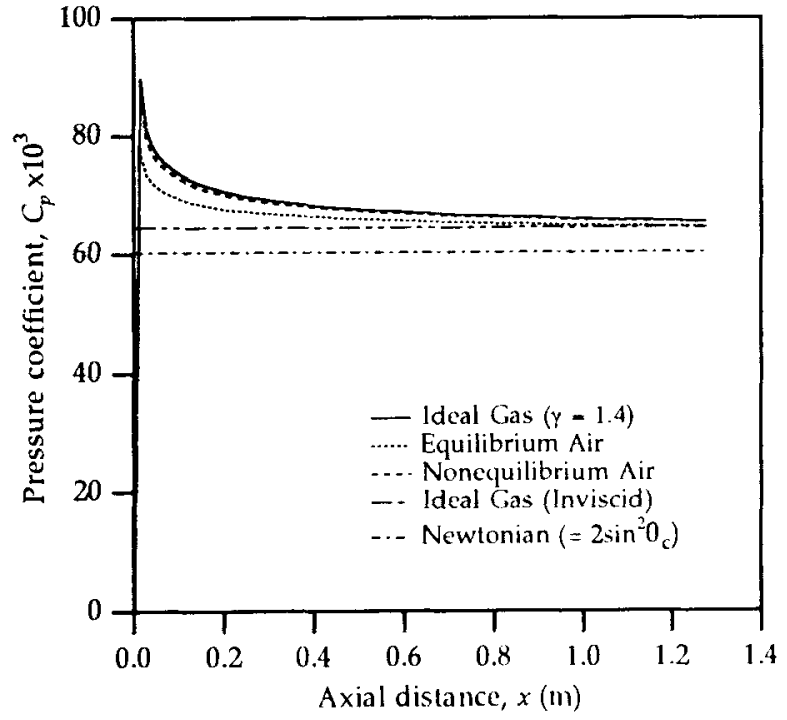

Figure 19. Effect of ideal, equilibrium and nonequilibrium air models on the computed surface pressure coefficient. The inviscid ideal gas value of $C_{p}$ is extrapolated from the tables of Sims (1964). 


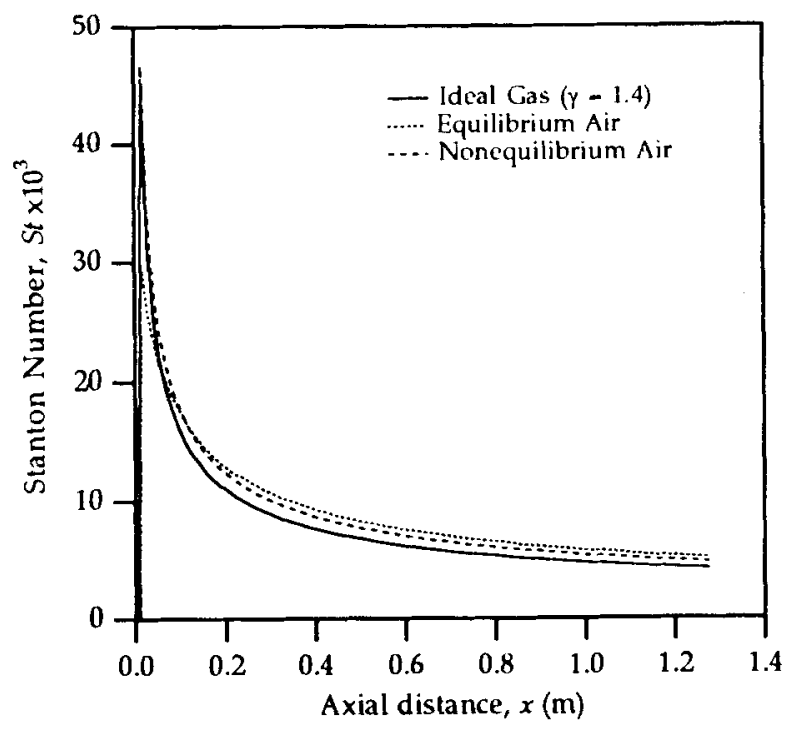

Figure 20. Effect of ideal, equilibrium and nonequilibrium air models on the computed Stanton number.

nearly the same irrespective of the gas model. The Newtonian value of the pressure coefficient is also shown along with the inviscid ideal gas value obtained from the tables of Sims (1964). Actually, the cone tables of Sims provide $C_{p}$ values for freestream Mach numbers ranging from 1.5 to 20.0 and for cone angles ranging from $2.5^{\circ}$ to $30^{\circ}$. The value corresponding to $M_{\infty}=25$ for a cone angle of $10^{\circ}$ was extrapolated from the tabulated values. The computed surface pressure coefficient for the ideal gas model asymptotes to the inviscid ideal gas value of Sims in the weak interaction zone.

The axial variation of the Stanton number ( $\mathrm{St}$ ) is shown in figure 20. The Stanton number is higher for the equilibrium case than the nonequilibrium case which, in turn, is higher than that for the ideal gas case.

The displacement thickness $\delta^{*}(\mathrm{~m})$ of a wall boundary layer is defined as:

$$
\delta^{*}=\int_{0}^{z_{h_{i}}}\left(1-\left(\rho u_{t}\right) / \rho_{e} u_{t_{\mathrm{e}}}\right) \mathrm{d} z_{b},
$$

where $z_{b}(\mathrm{~m})$ is measured along a line normal to the cone surface, $u_{t}(\mathrm{~m} / \mathrm{s})$ is the velocity tangential to the cone surface, and the subscript $e$ denotes edge values. In the present PNS code the grid lines are always normal to the body surface. Consequently, locating the boundary-layer edge is quite simple. The edge of the boundary layer is located (at every step) by using the specific total enthalpy $H$ of the gas. For an isothermal wall, the specific total enthalpy is dissipated in the viscous region close to the wall. The point $z_{b_{c}}$ at which the dissipation ceases, or equivalently where the total enthalpy reaches $98 \%$ of its freestream value, is chosen as the boundary-layer edge. See for instance the profiles of $H$ at $x=1.276 \mathrm{~m}$ shown in figure 21. Using the value of $z_{b_{e}}$ and (74), the displacement thickness is computed.

The axial variation of the displacement thickness of the boundary layer is shown in figure 22. There are two important features to be noted here. The first is that the axial variation of $\delta^{*}$ is shown only from about $x=0.2 \mathrm{~m}$. This is because there was difficulty in locating the boundary-layer edge near the tip of the cone. For a high Mach number and low Reynolds number flow, the viscous-inviscid interaction near the tip region is 


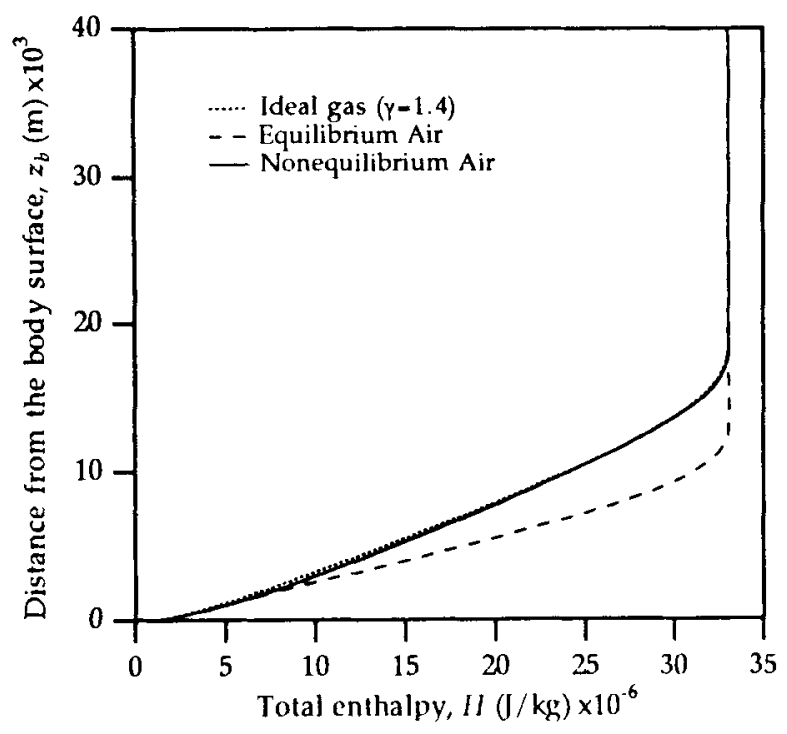

Figure 21. Profiles of total enthalpy at $x=1.276 \mathrm{~m}$. The total enthalpy is dissipated in the viscous layer close to the wall when the wall is isothermal. This profile helps in locating the boundary-layer edge which is then used in the computation of displacement thickness.

very strong. In fact there is a merger of the shock and viscous boundary layer making it difficult to define the boundary-layer edge. While the boundary layer in the equilibrium gas is considerably thinner, the boundary layers in the ideal and nonequilibrium gases are nearly of the same thickness. The value of viscosity for the nonequilibrium and ideal gas cases is not very different from each other except in the high-temperature region near the wall. Further, the rate at which the total enthalpy dissipates in the boundary layer is the same for both the ideal and nonequilibrium cases.

An important feature of hypersonic external flows is that the viscous shock layers are extremely thin. This was verified in the present computations through the shock standoff distance. The approximate shock standoff distance at every $x$ station was

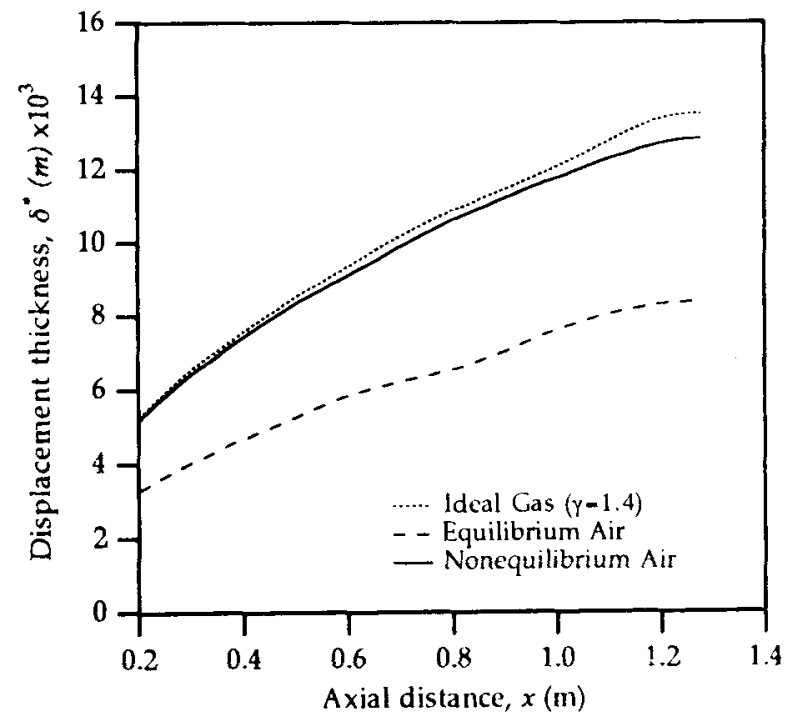

Figure 22. Axial variation of the computed displacement thickness for the ideal, equilibrium and nonequilibrium gas models. The computed data before $x=1.276 \mathrm{~m}$ are not reliable due to strong viscous-inviscid interaction which poses difficulties in locating the boundarylayer edge. 


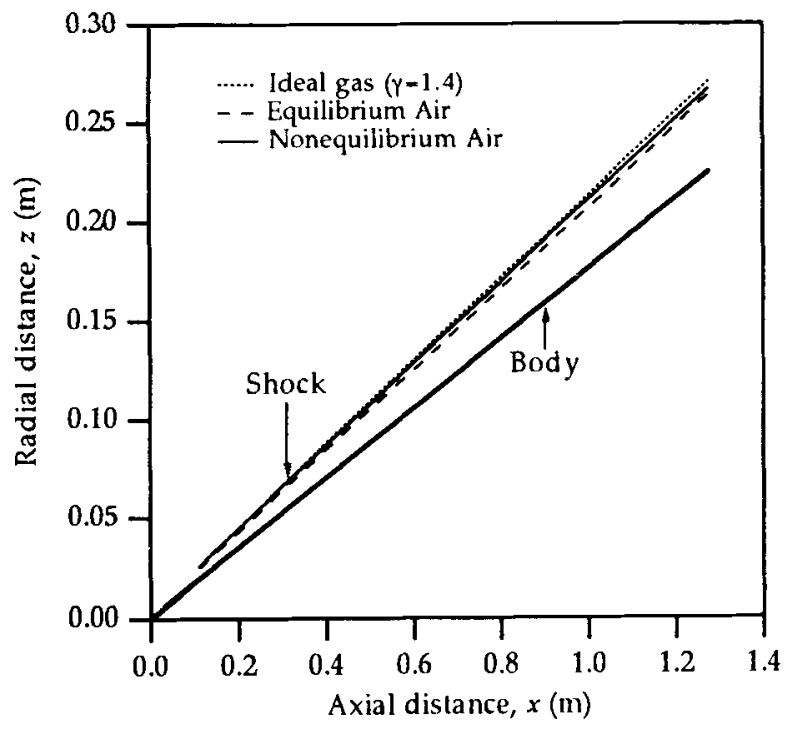

Figure 23. Comparison of shock shapes for ideal, equilibrium and nonequilibrium air models. The shock is "captured" as part of the solution. The shock is located by the peak in the pressure profile. Due to strong viscous-inviscid interaction it is not possible to locate the shock very accurately before $x=0 \cdot 1 \mathrm{~m}$.

determined by locating the peak point in the pressure profile at that station. Since the shock was not "fit" as an infinitesimally thin discontinuity but was captured, the pressure peak location was assumed to be a good measure. The shock standoff distance as a function of $x$ for the ideal, equilibrium, and chemical nonequilibrium air models is shown in figure 23. Again the axial variation is shown only from $x=0.1 \mathrm{~m}$ because distinguishing between the shock and boundary layer before this location is quite difficult. It is clearly seen that the shock angle (measured from the body) is quite small. Further, the shock standoff distance for equilibrium air is the smallest while that for ideal gas is the largest. This is because of $\bar{\gamma}$ varying between 1.4 for the ideal gas and $1 \cdot 18$ for equilibrium air.

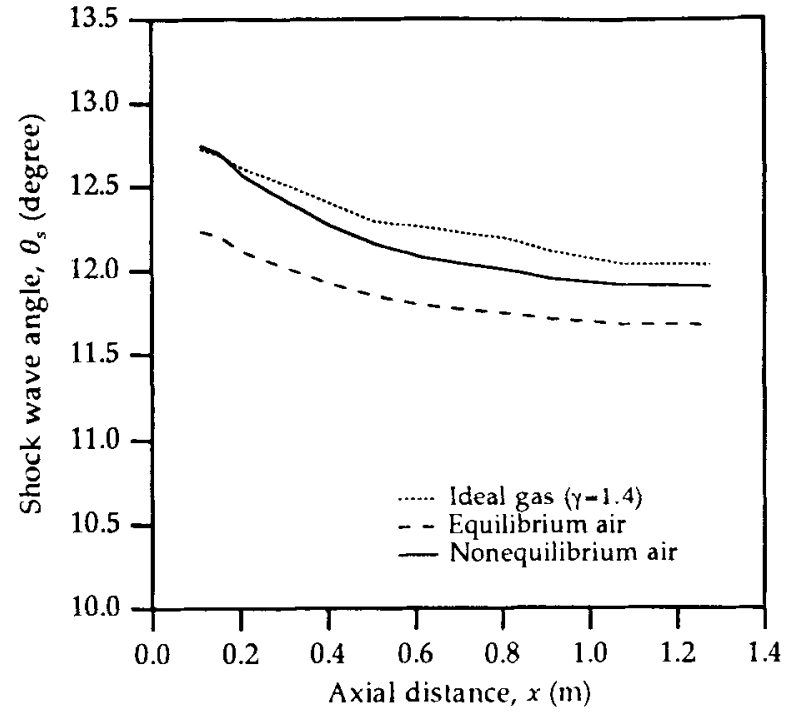

Figure 24. Approximate shock wave angles for ideal, equilibrium, and nonequilibrium air models. The displacement effect of the boundary layer causes the shock top curve initially (in the strong interaction zone) before it straightens out further downstream. 
To determine whether or not the outer shock was conical, the local inclination of the shock was computed. The axial variations of the local shock angle for the ideal, equilibrium and chemical nonequilibrium air models are shown in figure 24 . The shock inclination varies as a function of $x$ while asymptoting to a constant value due to the displacement effect of the boundary layer. The fairly thick boundary-layer at the leading edge pushes up against the shock thus altering the conical nature. As the inviscid-viscous interaction decreases downstream, the shock becomes nearly conical.

All these results presented here were obtained on an HCL Magnum Multi-RISC computer in the CTFD Division of NAL. The ideal gas calculations took $166 \mathrm{~s}$ of CPU time, the equilibrium air calculations took $204 \mathrm{~s}$ of CPU time, and the nonequilibrium gas calculations with the Liu-Vinokur model for species thermodynamic properties took about $580 \mathrm{~s}$ of CPU time.

\section{Concluding remarks}

The laminar hypersonic flow of air around a 10 cone has been successfully computed using a finite-difference algorithm. Three gas models have been considered - (1) ideal gas $(\gamma=1.4)$, (2) equilibrium air with curve-fits for thermodynamic and transport properties, and (3) nonequilibrium air with finite-rate chemistry. Based on the results of the calculations the following observations can be made.

- The numerical algorithm chosen for the present study is consistent, in the sense that the solution converges with diminishing grid spacing. This has been verified for both the ideal and nonequilibrium air cases.

- The choice of an unbiased differencing scheme such as central difference leads to poor definition of the captured shock. Artificial dissipation which is introduced in an ad hoc manner in such schemes is essential to stabilise the solution and get rid of the high-frequency errors. Low levels of artificial dissipation lead to "ringing" at the shock. Further, this artificial dissipation has to be increased with increasing grid size. The arbitrariness associated with artificial smoothing can be removed through the use of robust upwind methods - methods which bias the differencing in the direction of acoustic signal propagation (Lawrence 1991).

- The results of the calculations can change significantly depending on the choice of the gas model. The idealisation that the species lack an internal structure is not very good. However, for the test case chosen in the present study, the results obtained using the Blottner model (Blottner et al 1971) for nonequilibrium calculations are not significantly different from those obtained using a more accurate model developed by Liu \& Vinokur (1989). This is because the temperatures in the flowfield do not exceed $8000 \mathrm{~K}$ and below this temperature the thermodynamic properties of the species are nearly the same. However, the thermodynamic model of Liu and Vinokur is recommended for its accuracy above temperatures of $8000 \mathrm{~K}$.

- Some key features of hypersonic shock layers have been shown in the present study - thinness of the viscous shock layer, the displacement effect on the outer shock due to the boundary layer, the strong viscous-inviscid interaction near the nose of the cone, the effects of high temperatures in the shock layer etc. have been demonstrated.

Apart from the accuracy of the species thermodynamic properties, there are also issues associated with the accuracy of the species transport properties and reaction 
rates. The reaction rates and transport coefficients due to Blottner et al (1971) are fairly old and need to be updated. The sensitivity of the solutions to the accuracy of these parameters must be studied.

There is a paucity (at least in the public domain) of experimental results for hypersonic speeds and this makes the task of validation of a hypersonic code with high-temperature effects very difficult. To some extent, the aerothermodynamic environment encountered at hypersonic speeds can be created through a large shock tunnel (either combustion or free piston driven). This, however, does not currently exist in India.

In the present code, the block-tridiagonal solver is written for a general block of order $n$ and this solver does not use any pivoting (either partial or full) in the LU decomposition of the block and is thus quite fast. However, this solver is slower than the one that is "hardwired" for a particular block size. This disadvantage of the general solver has to be carefully weighed against the advantage of reusability that such a solver has to offer.

I thank the AR \& DB for support of this work under NAL Project No. CF-1-124. I also wish to thank Dr S S Desai for his encouragement. A portion of this work was presented at a Fluid Dynamics Colloquium at the Jawaharlal Nehru Centre of Advanced Scientific Research, Indian Institute of Science, Bangalore on September 22, 1993 for which I am grateful to Prof R Narasimha. Finally, I would like to thank the reviewers for their comments and suggestions.

\section{List of symbols}

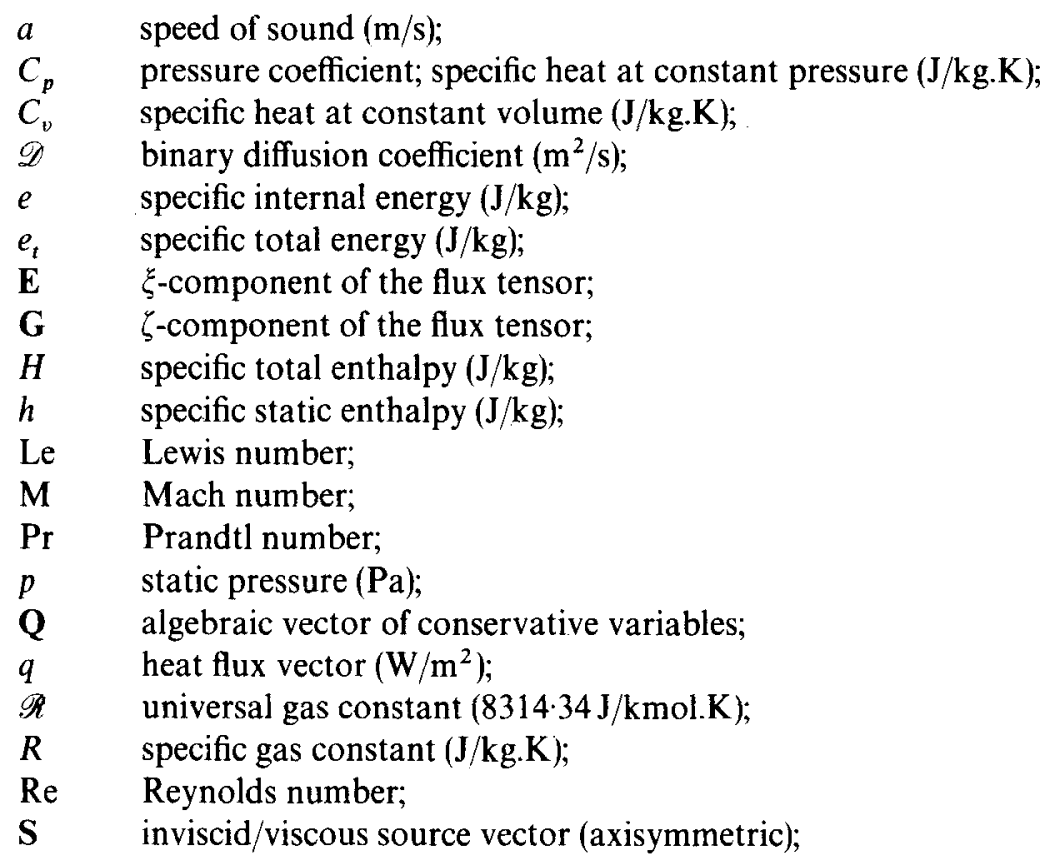




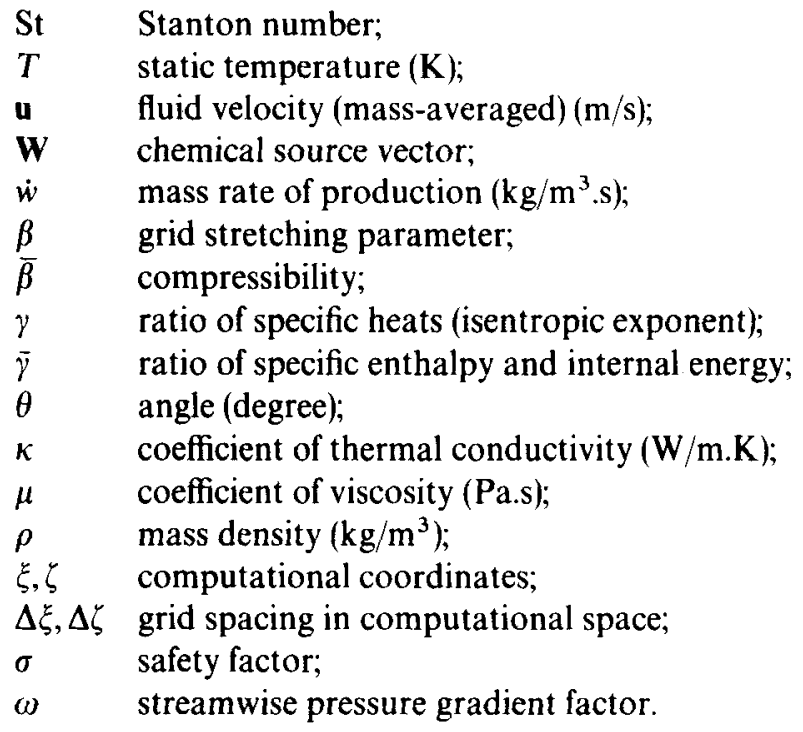

\section{Subscripts}

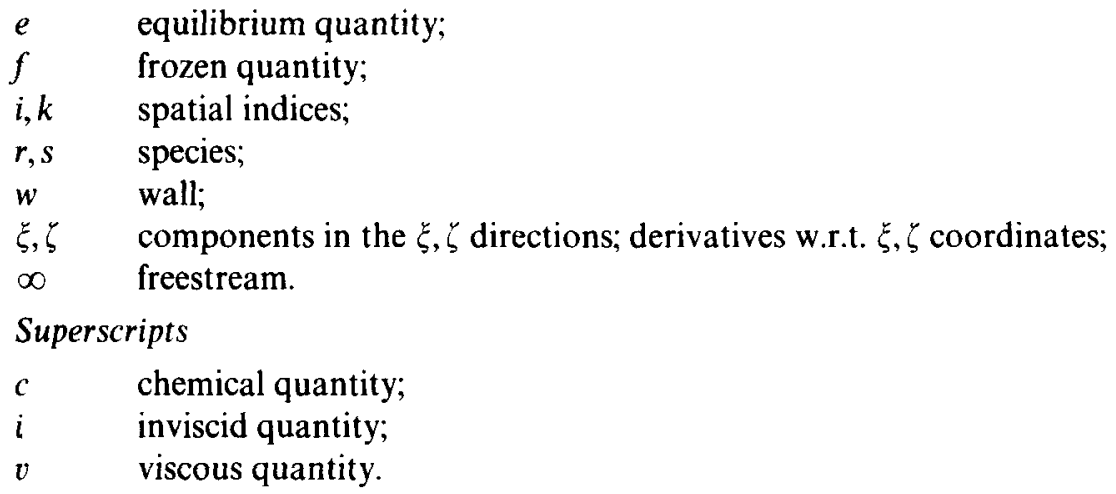

\section{References}

Anderson J D 1989 Hypersonic and high-temperature gas dynamics (New York: McGraw-Hill) Anderson D A, Tannehill J C, Pletcher R H 1982 Computational fuid dynamics (New York: McGraw-Hill)

Bailey H E 1967 Programs for computing equilibrium thermodynamic properties of gases. NASA TN D-3921

Blottner F G, Johnson M, Ellis M 1971 Chemically reacting viscous flow program for multi-component gas mixtures. Report no. SC-RR-70-754, Sandia Laboratories, Albuquerque, New Mexico

Cambier J-L, Prabhu D K 1992 Numerical simulations of nonequilibrium shock layers with highly efficient implicit schemes. AIAA paper no. 92-2973

Cambier J-L, Tokcarcik S, Prabhu D K 1992 Numerical simulations of unsteady flow in a hypersonic shock tunnel facility. AIAA paper no. $92-4029$

Eucken A 1913 On the heat conductivity, the specific heat, and the internal friction of gases. Phys. Zeit. 14: 324-332

Gordon S, McBride B J 1976 Computer program for calculations of complex chemical equilibrium compositions, rocket performance, incident and reflected shock, and ChapmanJouguet detonations. NASA SP-273 
Lawrence S L 1991 Parabolized Navier-Stokes methods for hypersonic flows. VKI Lecture Series in Computational Fluid Dynamics 1991-01 (Belgium: Von Karman Institute for Fluid Dynamics)

Liu Y, Vinokur M 1989 Equilibrium gas flow computations: I. Accurate and efficient calculation of equilibrium properties. AIAA paper no. 89-1736

Park C 1990 Nonequilibrium hypersonic aerothermodynamics (New York: John Wiley)

Prabhu D K 1987 A new parabolized Navier-Stokes code for chemically reacting flow fields $\mathrm{Ph} D$ Dissertation, Iowa State University, Ames, IA

Prabhu D K 1993 High-temperature effects in hypersonic flows. NAL PD CF-9319, National Aerospace Laboratories, Bangalore

Prabhu D K, Tannehill J C 1986 Numerical solution of Space Shuttle Orbiter flowfield including real-gas effects. J. Spacecr. Rockets 23: 264-272

Prabhu D K, Tannehill J C, Marvin J G 1988 A new PNS code for chemical nonequilibrium flows. AIAA J. 26: 808-815

Prabhu D K, Subramanian N R, Saxena S K 1993 Hypersonic viscous flow computations using parabolized Navier-Stokes equations. NAL PDCF-9303, National Aerospace Laboratories, Bangalore

Rakich J V, Venkatapathy E, Tannehill J C, Prabhu D 1984 Numerical solution of Space Shuttle Orbiter flowfield. J. Spacecr. Rockets 21: 9-15

Rubin S G, Tannehill J C 1992 Parabolized/reduced Navier-Stokes computational techniques. Annu. Rev. Fluid Mech. 24: 117-144

Schiff L B, Steger J L 1979 Numerical simulations of steady supersonic viscous flows. AIAA paper no. 79-0130

Sims J L 1964 Tables for supersonic flow around right circular cones at zero angle of attack. NASA SP-3004

Srinivasan S, Tannehill J C, Weilmuenster K J 1987a Simplified curve fits for the thermodynamic properties of equilibrium air. NASA RP-1181

Srinivasan S, Tannehill J C, Weilmuenster K J 1987b Simplified curve fits for the transport properties of equilibrium air. NASA CR-178411

Tannehill J C, Venkatapathy E, Rakich J V 1982 Numerical solution of supersonic viscous flow over blunt delta wings. AIAA J. 20: 203-210

Vigneron Y C, Rakich J V, Tannehill J C 1978 Calculation of supersonic flow over delta wings with sharp subsonic leading edges. AIAA paper no. 78-1137

Wilke C R 1950 A viscosity equation for gas mixtures. J. Chem. Phys. 18: 517-519 